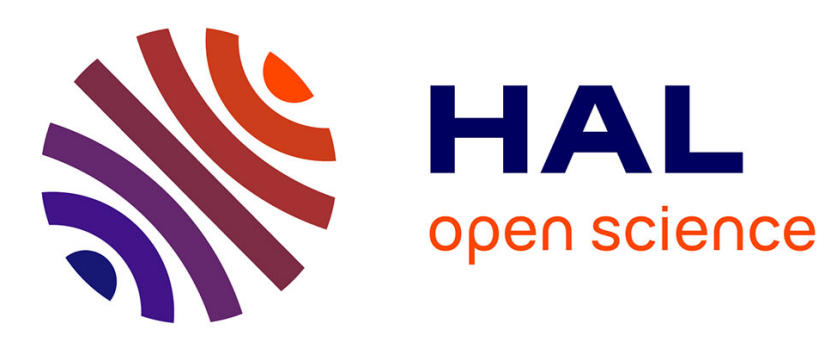

\title{
Extension of SEA model to subsystems with non-uniform modal energy distribution
}

\author{
Laurent Maxit, Jean-Louis Guyader
}

\section{To cite this version:}

Laurent Maxit, Jean-Louis Guyader. Extension of SEA model to subsystems with non-uniform modal energy distribution. Journal of Sound and Vibration, 2003, 265, pp.337-358. 10.1016/S0022460X(02)01459-1 . hal-01162178

\section{HAL Id: hal-01162178 https://hal.science/hal-01162178}

Submitted on 9 Jun 2015

HAL is a multi-disciplinary open access archive for the deposit and dissemination of scientific research documents, whether they are published or not. The documents may come from teaching and research institutions in France or abroad, or from public or private research centers.
L'archive ouverte pluridisciplinaire HAL, est destinée au dépôt et à la diffusion de documents scientifiques de niveau recherche, publiés ou non, émanant des établissements d'enseignement et de recherche français ou étrangers, des laboratoires publics ou privés. 


\title{
EXTENSION OF SEA MODEL TO SUBSYSTEMS WITH NON \\ UNIFORM MODAL ENERGY DISTRIBUTION
}

\author{
L. MAXIT and J.-L. GUYADER \\ INSA de Lyon (National Institute of Applied Sciences) \\ Vibrations - Acoustic Laboratory \\ 25 bis, avenue Jean Capelle \\ 69621 VILLEURBANNE Cedex - FRANCE \\ Accepted for publication \\ Journal of Sound and Vibration
}

Headline : SEA reformulation

$\underline{\text { Address for proofs: }}$

INSA de Lyon (National Institute of Applied Sciences)

L.V.A. (Vibrations - Acoustic Laboratory)

Bâtiment Antoine de Saint Exupéry

25 bis, avenue Jean Capelle

69621 VILLEURBANNE Cedex - FRANCE

+33472438080

+33472438712(fax)

maxit@1va.insa-lyon.fr, guyader@1va.insa-lyon.fr

Pages $\quad 42 \quad$ (including Figure captions, Figures, Tables)

Figures $\quad 16$

Tables 2

Footnotes 0 


\section{Summary:}

In order to enlarge the application field of Statistical Energy Analysis (SEA), a reformulation is proposed. The model described here, Statistical modal Energy distribution Analysis (SmEdA), does not assume equipartition of modal energies contrary to classical SEA.

Theoretical derivations are based on dual modal formulation described in [1,2] for the general

case of coupled continuous elastic systems. Basic SEA relations describing power flow exchanged by two oscillators are used to obtain modal energy equations. They permit to determine modal energies of coupled subsystems from the knowledge of modes of uncoupled subsystems. The link between SEA and SmEdA is established and render possible to mix the two approaches: SmEdA for subsystems where equipartition is not verified and SEA for other subsystems.

Three typical configurations of structural couplings are described for which SmEdA improves energy prediction compared to SEA: (a) coupling of subsystems with low modal overlap. (b) coupling of heterogeneous subsystems. (c) case of localised excitations.

The application of the proposed method is not limited to academic structures, but could easily be applied to complex structures by using finite element method (FEM). In this case, FEM are used to calculate the modes of each uncoupled subsystems; these data are then used in a second step to determine modal coupling factors necessary to SmEdA to modelise the coupling. 


\section{INTRODUCTION}

Statistical Energy Analysis (SEA) [3-5] has been developed to predict noise and vibration transmission through complex structures at medium and high frequencies. In SEA, the built-up structure is subdivided into a number of subsystems and the vibration response within each subsystem is characterized by the subsystem energy. Derivation of SEA is based on several assumptions (see [6]), and its validity domain is not easy to establish even if many studies addressed this point [7-29]. Let us first present some cases, where SEA give poor prediction.

Yap and Woodhouse [7] have studied the influence of damping on the quality of SEA results. Equivalent Coupling Loss Factor (CLF) was obtained from numerical simulations on beam and plate coupling. They were shown to depend strongly on damping, whereas CLF obtained classically by the wave approach were independent of damping. For weakly damped system, equivalent CLFs are proportional to Damping Loss Factors (DLF), and values are lower than those given by the wave approach, i.e. wave approach leads to overestimate the energy transfer. The authors attributed the strong dependency to damping to the fact that energy equipartition does not hold when damping is low.

For three plates coupled in U shape, Fredo [8] showed that indirect coupling between first and third plate can be significant if damping is weak. The indirect coupling was attributed to the nonobservance of one or more SEA assumptions. Previously, Finnveden had already shown in the case of three coupled elements [9] that SEA seriously overestimates the flow of energy when damping loss factors are small.

The coupling of two irregular plates has been studied by Mace et al. [10], [11]. For large damping, the response is independent of the shape of the plate and wave estimate of CLF gives accurate predictions. Contrary to light damping, the transmission depends significantly on the specific geometry of each plate; and the power transmitted is often substantially less than that predicted by SEA.

Ming et Pan studied the accuracy of SEA results on coupled plates [12]. They noticed that two parameters influence the quality result : the geometric mean of the modal overlap factors and the number of resonant modes in the frequency band of interest. At low frequency, where there are few modes, SEA results are poor and exact results present a high sensitivity to the position of the excitation point. Increasing frequency, modal overlap factors and modes number increase ; then 
SEA results are better and the sensitivity to the position of excitation decreases (see also [13]). A previous work of Fahy and Mohammed [14] on beam-plate coupling cases gave similar conclusions. When the modal overlap factor is much less than unity, SEA overestimates energy transfer.

From analytical calculations of the power exchanged by two coupled one dimensional subsystems, Mace $[15,16]$ proposed the $\gamma$ parameter to characterize the coupling strength. This parameter is defined by the ratio of the transmission factor to the product of the two modal overlap in both connected subsystem. When $\gamma$ is small, the coupling is called weak, i.e. classical SEA is valid. Finnveden $[17,18]$ deduced the same coupling strength criterion by investigating the ensemble averaged power flow in a three elements structure.

One can conclude that SEA has serious difficulties to predict energy transfer when SEA assumptions are not verified in at least one subsystem. This was the key point of our interest in developing a refinement of SEA, based on not so restrictive assumptions.

Langley [19, 20] proposed an extension of SEA called Wave Intensity Analysis (WIA). Classical SEA assumed that the vibrational wavefield in each subsystem is diffuse. In some cases (not explicitly defined by the author), this assumption can be not fulfilled leading SEA to yield poor estimates of vibrational responses. In WIA, the directional dependency of the vibrational wavefield in each subsystem is derived using Fourier series. When the first term of the Fourier serie is only considered, WIA is equivalent to SEA. Adding terms in the series improve predictions for plate assemblies [19-22].

The study presented in this paper is also based on the reformulation of SEA with less restrictive assumptions. The goal is to extend the validity of the model to cases where classical SEA was seen unsatisfactory. The assumption that one wishes to remove in this paper is equipartition of energy. The approach, based on the dual modal formulation proposed in [1,2] take into account the modal energies distribution of each subsystem. The present model is called SmEdA (Statistical modal Energy distribution Analysis) and can be seen as a refinement of traditional SEA.

\section{DUAL MODAL FORMULATION}

The dual modal formulation described in [1] for the general case of coupled continuous elastic systems is based on a dual displacement-stress formulation and two kinds of subsystem modes : 
uncoupled-free modes and uncoupled-blocked modes. The results which constitute the base of SmEdA model are summarised here. (For more details, see references [1,2].)

\subsection{COUPLING OF TWO CONTINUOUS MECHANICAL SYSTEMS}

Two mechanical systems are considered. They are rigidly coupled on a surface $S_{\text {Coupling }}$ as shown in figure 1. Both systems are excited by random, ergodic excitations of band limited white noise type and the material of each subsystem is supposed to be linear elastic and have viscous damping.

Subsystem 1 is described by displacement vector $W_{i}(M, t)$ and subsystem 2 by stress tensor $\sigma_{i j}\left(M^{\prime}, t\right)$ where $i$ and $j=1,2,3, t$ is time, and $M$ (resp. $M^{\prime}$ ) denotes point of subsystem 1 (resp. subsystem 2). According to the dual modal formulation, subsystem 1 is described by modes of the uncoupled-free subsystem (null stresses on $S_{\text {Coupling }}$ ) and subsystem 2 by modes of the uncoupled-blocked subsystem (null displacements on $S_{\text {Coupling }}$ ). (see figure 2.)

Expanding displacements of subsystem 1 and stresses of subsystem 2, and assuming responses controlled by resonant contributions, gives :

$$
\begin{aligned}
& W_{i}(M, t)=\sum_{n=1}^{N_{1}} a_{n}(t) \tilde{W}_{i}^{n}(M), \\
& \sigma_{i j}\left(M^{\prime}, t\right)=\sum_{s=1}^{N_{2}} b_{s}(t) \tilde{\sigma}_{i j}^{s}\left(M^{\prime}\right),
\end{aligned}
$$

where :

- $a_{n}(t), b_{s}(t)$ are modal amplitudes for subsystem 1 and subsystem 2, respectively ;

- $\tilde{W}_{i}^{n}(M)$ are displacement mode shapes of subsystem 1 ;

- $\tilde{\sigma}_{i j}^{s}\left(M^{\prime}\right)$ are stress mode shapes of subsystem 2, and ;

$-N_{1}, N_{2}$ are the number of resonant modes of subsystem 1 and subsystem 2, respectively.

With the change of modal variable,

$$
b_{q}(t)=\dot{c}_{q}(t)
$$

the modal equations given by the dual modal formulation (see [1]) is : 


$$
\left\{\begin{array}{l}
\ddot{a}_{p}(t)+\Delta_{p} \dot{a}_{p}(t)+\left(\omega_{p}\right)^{2} a_{p}(t)+\frac{1}{M_{p}} \sum_{m=1}^{N_{2}} \dot{c}_{m}(t) \mathbf{W}_{p m}=\frac{F_{p}}{M_{p}}, \quad \forall p \in\left[1, \ldots, N_{1}\right], \\
\ddot{c}_{q}(t)+\Delta_{q} \dot{c}_{q}(t)+\left(\omega_{q}\right)^{2} c_{q}(t)-\frac{1}{\left(\omega_{q}\right)^{2} M_{q}} \sum_{r=1}^{N_{1}} \dot{a}_{r}(t) \mathbf{W}_{r q}=\frac{F_{q}}{\left(\omega_{q}\right)^{2} M_{q}}, \quad \forall q \in\left[1, \ldots, N_{2}\right],
\end{array}\right.
$$

where :

- $\Delta_{p}, \Delta_{q}$ are modal damping bandwidths of each subsystem;

- $\omega_{p}, \omega_{q}$ are natural angular frequencies of uncoupled subsystems;

- $M_{p}, M_{q}$ are modal masses;

- $F_{p}, F_{q}$ are generalised modal forces, and;

- $\mathbf{W}_{p q}$ are interaction modal works yielded for each couple of modes $(p, q)$ by :

$$
\mathbf{W}_{p q}=\int_{S_{\text {Coupling }}} \tilde{W}_{i}^{p} \tilde{\sigma}_{i j}^{q} n_{j}^{2} d S
$$

and $n_{j}^{2}$ are components of the outer normal vector of the volume occupied by subsystem 2 .

This system of equations describes the forced response of the coupled subsystems from the amplitudes of modes of the uncoupled subsystems.

The form of these equations allows us to interpret modes interactions as oscillators with gyroscopic couplings (see figure 3). It is pointed out that a mode of one subsystem is coupled to the modes of the other subsystem but is not directly coupled with the other modes of the subsystem to which it belongs. This configuration of mode coupling is exactly the one that supposes SEA.

\section{REFORMULATION OF SEA MODEL WITHOUT EQUIPARTITION ASSUMPTION}

One proposes in this section to reformulate SEA model without taking into account equipartition of energy.

\subsection{MODAL ENERGY EQUATIONS}

Let us consider mode $p$ of subsystem 1. Its equation of motion is given in (4). The principle of conservation of energy applied to this mode gives :

$$
\Pi_{i n j}^{p}=\Pi_{d i s s}^{p}+\sum_{q=1}^{N_{2}} \Pi_{p q}, \quad \forall p \in\left[1, \ldots, N_{1}\right],
$$

where: 
- $\Pi_{i n j}^{p}$ is time-averaged injected power by the generalized force $F_{p}$;

- $\Pi_{\text {diss }}^{p}$ is time-averaged dissipated power by internal damping of mode $p$, and ;

- $\sum_{q=1}^{N_{2}} \Pi_{p q}$ is time-averaged power flow exchanged by mode $p$ with the modes of subsystem 2 The injected power into mode $p$ by external excitation is, either dissipated by internal damping of the mode or exchanged with modes of subsystem 2. Let us estimate the different powers appearing in this equation :

- Evaluating $\Pi_{i n j}^{p}$ from the power injected relation established for an oscillator excited by a white noise force ([2]) gives:

$$
\Pi_{i n j}^{p}=\frac{\pi}{4 M_{p}} \bar{S}_{F_{p}},
$$

where $\bar{S}_{F_{p}}$ is the power spectral density of the generalised force expressed in $\mathrm{N}^{2} /(\mathrm{rad} / \mathrm{s})$.

- The power dissipated by internal damping of an oscillator (see [5]) can be related to its total energy by expression (9):

$$
\Pi_{\text {diss }}^{p}=\omega_{p} \eta_{p} E_{p},
$$

where $E_{p}$ is the time averaged energy of mode $p$, and $\eta_{p}$ is the modal damping factor $\left(\Delta_{p}=\omega_{p} \eta_{p}\right)$.

- To evaluate the power exchanged by mode $p$ of subsystem 1 with mode $q$ of subsystem 2 , one isolates these two modes in the modal equations of motion (4) and (5) :

$$
\left\{\begin{array}{l}
\ddot{a}_{p}(t)+\Delta_{p} \dot{a}_{p}(t)+\left(\omega_{p}\right)^{2} a_{p}(t)+\sqrt{\frac{\left(\omega_{q}\right)^{2} M_{q}}{M_{p}}} \gamma_{p q} \dot{c}_{q}(t)=L_{1 p q}(t), \\
\quad \forall(p, q) \in\left(\left[1, N_{1}\right],\left[1, N_{2}\right]\right), \\
\ddot{c}_{q}(t)+\Delta_{q} \dot{c}_{q}(t)+\left(\omega_{q}\right)^{2} c_{q}(t)-\sqrt{\frac{M_{p}}{\left(\omega_{q}\right)^{2} M_{q}}} \gamma_{p q} \dot{a}_{p}(t)=L_{2 q p}(t),
\end{array}\right.
$$

where : $L_{1 p q}(t)=\frac{F_{p}(t)}{M_{p}}-\sum_{\substack{r=1 \\ r \neq q}}^{N_{2}} \frac{\mathbf{W}_{p r}}{M_{p}} \dot{c}_{r}(t), L_{2 q p}(t)=\frac{F_{q}(t)}{\left(\omega_{q}\right)^{2} M_{q}}+\sum_{\substack{m=1 \\ m \neq p}}^{N_{1}} \frac{\mathbf{W}_{m q}}{\left(\omega_{q}\right)^{2} M_{q}} \dot{a}_{m}(t)$, and,

$\gamma_{p q}=\frac{\mathbf{W}_{p q}}{\sqrt{M_{p}\left(\omega_{q}\right)^{2} M_{q}}}$

Supposing as classically done in SEA that the interaction forces $L_{1 p q}(t)$ and $L_{2 q p}(t)$ are uncorrelated white noise forces, the basic SEA relation established by Sharton and Lyon [30] can be used: 


$$
\Pi_{p q}=\omega_{c} \eta_{p q}\left(E_{p}-E_{q}\right)
$$

where $\omega_{c}$ is the central angular frequency of the band of interest, and, $\eta_{p q}$ is called the modal coupling loss factor (see [1]). It is a function of natural angular frequencies, $\omega_{p}, \omega_{q}$; modal masses, $M_{p}, M_{q} ;$ modal bandwidths, $\Delta_{p}, \Delta_{q} ;$ and interaction modal works, $\mathbf{W}_{p q}$ :

$$
\eta_{p q}=\frac{\left(\mathbf{W}_{p q}\right)^{2}}{\omega_{c} M_{p}\left(\omega_{q}\right)^{2} M_{q}}\left(\frac{\left(\Delta_{p}\left(\omega_{q}\right)^{2}+\Delta_{q}\left(\omega_{p}\right)^{2}\right)}{\left(\left(\omega_{p}\right)^{2}-\left(\omega_{q}\right)^{2}\right)^{2}+\left(\Delta_{p}+\Delta_{q}\right)\left(\Delta_{p}\left(\omega_{q}\right)^{2}+\Delta_{q}\left(\omega_{p}\right)^{2}\right)}\right) .
$$

Introducing (8), (9), and (12) in (7), one obtains power balance equations (14) for mode $p$ of subsystem 1:

$$
\Pi_{i n j}^{p}=\omega_{p} \eta_{p} E_{p}+\sum_{q=1}^{N_{2}} \omega_{c} \eta_{p q}\left(E_{p}-E_{q}\right), \quad \forall p \in\left[1, \ldots, N_{1}\right]
$$

In the same way, energy balance equation of mode $q$ of subsystem 2 can be written:

$$
\Pi_{i n j}^{q}=\omega_{q} \eta_{q} E_{q}+\sum_{p=1}^{N_{1}} \omega_{c} \eta_{p q}\left(E_{q}-E_{p}\right), \quad \forall q \in\left[1, \ldots, N_{2}\right] .
$$

The relations (14) and (15) constitute a linear system of modal energies of subsystems 1 and 2 :

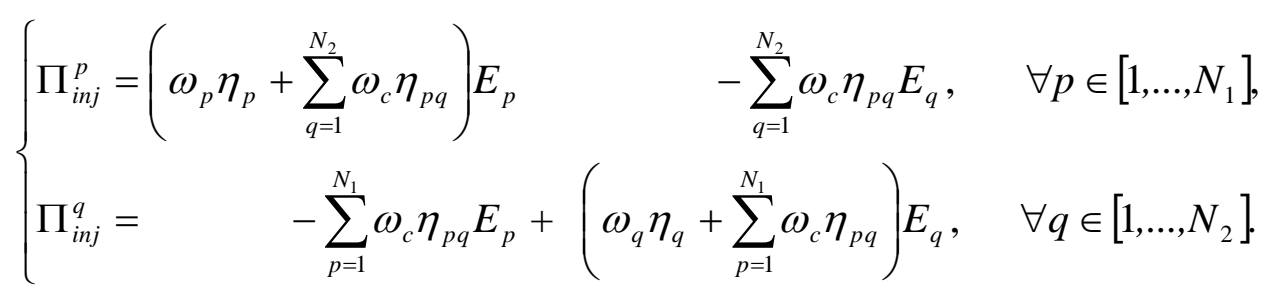

The total energy of each subsystem can be finally obtained by adding modal energies :

$$
E_{1}=\sum_{p=1}^{N_{1}} E_{p}, \quad E_{2}=\sum_{q=1}^{N_{2}} E_{q}
$$

where $E_{1}$ (resp. $E_{2}$ ) is the time-averaged total energy of subsystem 1 (resp. subsystem 2).

The model attached to the modal energy equations (16) is called SmEdA (Statistical modal Energy distribution Analysis). The application of this model is not limited to academic structures, but can be apply to complex structures by using finite element method (FEM). As described in [1, 31] to evaluate CLFs, FEM can be used to calculate the uncoupled subsystems modes which permit to determine the modal coupling loss factors. 
In the next section, the link between SmEdA and SEA will be established.

\subsection{RELATIONS BETWEEN SEA AND SMEDA}

In classical SEA, modal energy equipartition is assumed and permits to restrict the $N_{l}$ degree of freedom (DOF) of subsystem 1 and the $N_{2}$ DOF of subsystem 2 to only two DOF, one per subsystem. Introducing equipartition relation (18),

$$
\begin{aligned}
& E_{p}=\frac{E_{1}}{N_{1}}, \forall p \in\left[1, N_{1}\right], \\
& E_{q}=\frac{E_{2}}{N_{2}}, \forall q \in\left[1, N_{2}\right],
\end{aligned}
$$

in the modal energy equations (16), and adding energy balance equations of subsystem 1 modes (resp. subsystem 2 modes), gives us the standard SEA equation (19) :

$$
\left\{\begin{array}{l}
\Pi_{i n j}^{1}=\omega_{c} \eta_{1} E_{1}+\omega_{c} \eta_{12}\left(E_{1}-\frac{N_{1}}{N_{2}} E_{2}\right) \\
\Pi_{i n j}^{2}=\omega_{c} \eta_{2} E_{2}+\omega_{c} \eta_{12}\left(\frac{N_{1}}{N_{2}} E_{2}-E_{1}\right)
\end{array}\right.
$$

where $\Pi_{i n j}^{1}=\sum_{p=1}^{N_{1}} \Pi_{i n j}^{p}\left(\right.$ resp. $\left.\Pi_{i n j}^{2}=\sum_{q=1}^{N_{2}} \Pi_{i n j}^{q}\right)$ represents the power injected by external sources in subsystem 1 (resp. subsystem 2), and, $\eta_{12}$ is the SEA coupling loss factor given by :

$$
\eta_{12}=\frac{1}{N_{1}} \sum_{p=1}^{N_{1}} \sum_{q=1}^{N_{2}} \eta_{p q} .
$$

In some practical applications, equipartition assumption can be fulfilled by some subsystems but not by the others. In these situations, it will be interesting to mix SEA and SmEdA: classical SEA being used for subsystems where equipartition is valid and SmEdA for the others. To precise this point, let us consider the case of two subsystems, assume equipartition valid for subsystem 1 and not for subsystem 2; equation (16) writes:

$$
\left\{\begin{array}{l}
\Pi_{i n j}^{1}=\omega_{c}\left(\eta_{1}+\eta_{12}\right) E_{1} \quad-\omega_{c} \sum_{q=1}^{N_{2}}\left(\sum_{p=1}^{N_{1}} \eta_{p q}\right) E_{q}, \\
\Pi_{i n j}^{q}=-\frac{\omega_{c}}{N_{1}}\left(\sum_{p=1}^{N_{1}} \eta_{p q}\right) E_{1}+\left(\omega_{q} \eta_{q}+\omega_{c} \sum_{p=1}^{N_{1}} \eta_{p q}\right) E_{q}, \quad \forall q \in\left[1, \ldots, N_{2}\right]
\end{array}\right.
$$

The unknowns of these equations are the total energy of subsystem 1 and the $N_{2}$ modal energies of subsystem 2. It is thus possible to only apply SmEdA for the subsystem where equipartition is not 
achieved, and to use classical SEA for the other subsystem. (Application to four coupled plates is proposed in [32]).

\section{SOME EXAMPLES}

Three typical cases where equipartition is not achieved are shown in this section: (a) coupling of subsystems with low modal overlap; (b) coupling of heterogeneous subsystems; (c) case of localised excitation.

The following examples are based on beam and plate couplings in order to simplify the calculation. However, it should not be seen as a limit of the approach; the application to complicated subsystems could be achieved, thanks to finite element models, in a straight forward manner.

In what follows, one notes by: SmEdA, the present approach; SEA ${ }^{\mathrm{DMF}}$, the SEA approach considering CLFs estimated by equation (13), i.e. the dual modal formulation; SEA ${ }^{\text {wave }}$, the SEA approach considering CLFs estimated by the classical wave approach (see [3]).

\subsection{COUPLING OF SUBSYSTEMS WITH LOW MODAL OVERLAP}

\subsubsection{Two subsystems}

Two pinned-pinned beams coupled rigidly at one end as shown figure 4 are considred. $L_{\alpha}, b_{\alpha}, h_{\alpha}$, $E_{\alpha}, \rho_{\alpha}$ are, respectively, length, width, thickness, Young's modulus, mass density of beam $\alpha$. The subsystem boundary conditions are simply supported at both ends for beam 1 and, clamped simply supported for beam 2. Rigid coupling is assumed that is to say continuity of angular rotations and flexural moments, thus, the interaction modal works $\mathbf{W}_{p q}$ are expressed by :

$$
\mathbf{W}_{p q}=\tilde{\theta}_{z}^{p} \tilde{\mathbf{M}}_{f}^{q}
$$

where:

$-\tilde{\theta}_{z}^{p}$ is the $p$ th mode angular rotation at the junction for beam 1, and;

- $\tilde{\mathbf{M}}_{f}^{q}$ is the $q$ th mode bending moment at the junction for beam 2 .

In the following, only beam 1 is excited in the normalised octave band of central frequency $1000 \mathrm{hz}$. The driving force is a 'rain on the roof' type, thus, power spectral densities of generalised forces (see equation (8)) are constant whatever the modes. In order to compare with classical calculations, solution based on wave decomposition for pure tone excitation was used, then frequency averaging 
of energy was done. Lastly, to approximate rain on the roof excitation, beam energies obtained for 20 excitation points randomly distributed over beam 1 were averaged.

Beam lengths were chosen for having sufficient modes resonant in the excited band (10 modes for beam 1 and 9 modes for beam 2).

Figure 5 shows beam energies ratio $E_{2} / E_{1}$ versus geometric mean modal overlap. The geometric mean modal overlap factor, $\bar{M}$ is given by:

$$
\bar{M}=\sqrt{M_{1} M_{2}},
$$

where:

- $M_{\alpha}$ is beam $\alpha$ modal overlap: $M_{\alpha}=\omega_{c} \eta_{\alpha} n_{\alpha}, \alpha \in[1,2]$;

- $\omega_{c}$ is the central angular frequency of the excited beam;

- $\eta_{\alpha}$ is the damping loss factor, and;

- $n_{\alpha}$ is the modal density of beam $\alpha$ (see [3]).

SmEdA results agree with classical calculation for any geometric mean modal overlap whereas SEA $^{\text {DMF }}$ gives poor estimates when $\bar{M}<0.1$. It can also be noticed that $\mathrm{SEA}^{\text {wave gives }}$ approximately the same results that $\mathrm{SEA}^{\mathrm{DMF}}$.

Poor prediction of SEA ${ }^{\mathrm{DMF}}$ suggests that equipartition is not achieved when modal overlap is weak. This point is confirmed in figure 6 where the distribution of modal energies of the non-excited beam is presented for four different values of the geometric mean modal overlap. For the strongest value, equipartition is quite fulfilled explaining that $\mathrm{SEA}^{\mathrm{DMF}}$ gives good results. For the lowest value (figure 6 (a)), there is a large disparity between modal energies: the $4^{\text {th }}$ mode in the frequency band considered largely dominates the response. In this case, SEA ${ }^{\mathrm{DMF}}$ gives poor results. However, strong disparities of modal energies do not lead systematically to a bad estimation of energies by SEA. Comparing figures 6 (a) and 6 (b), disparities of modal energies are similar but SEA results come out in a very different place in the two cases: through the middle in (b), but through the peak in (a). In case (a), SEA overestimates largely energy transfer; whereas in case (b); SEA prediction is reasonably good (see figure (5)).

Modal energy disparities are due to frequency coincidence that has a significant role in the modal couplings when modal overlap factors are less than one (see expression (13)); some modes can be strongly coupled whereas other ones are much less. It is shown figure 7 where modal coupling loss factors are plotted. When damping is low (figure 7, (a)), the coupling between the two beams are dominated by the interaction between the $4^{\text {th }}$ mode of beam 1 and the $4^{\text {th }}$ mode of beam 2 (due to 
close natural frequencies); then equipartition is not achieved (see figure 6, (a)). On the other hand, when the mean geometric modal overlap factor is equal or greater than one (see figure 7, (c,d)), all modes of beam 2 are strongly coupled to at least one mode of beam 1, in this case equipartition is achieved (see figure 6, (c,d)).

Several studies [7,12,14,29] have shown, as it can be observed here, that classical SEA overestimates the energy transfer between subsystems when modal overlap factors are much less than one. The present results demonstrate that, in this case, modal equipartition is not fulfilled and is responsible of the poor prediction of SEA. This was previously mentioned in reference [7].

The $\gamma$ factor was proposed separately by Mace [16] and Finnveden [17] as an indicator of the coupling strength of two subsystems. When $\gamma$ is much less than one, the coupling between two subsystems is called weak, i.e. classical SEA is valid.

$$
\gamma=\frac{\tau_{12}}{2 \pi^{2} M_{1} M_{2}}
$$

where $\tau_{12}$ is the transmission factor.

Table 1 presents the $\gamma$ factor (25) for different cases treated in figure 5. It can be observed that this criterion indicates well the validity of SEA; namely when $\gamma>1$, SEA $^{\text {wave }}$ fails, but SmEdA still permits to predict the energy flow and appears as alternative to SEA for strong coupling.

Another example, based on plate coupling was presented in reference [2]. Because the coupling effects are distributed along a line, spatial coincidence of mode shapes has a significant role and can lead several modes of the non excited subsystem, to be uncoupled with modes of the excited subsystem. This increases the disparity of the distribution of modal energies, SEA can then overestimate energy transfer even if geometric mean modal overlap is equal or greater than one, whereas SmEdA gives good results.

\subsubsection{Multiple-subsystems}

Now, let us consider a structure composed of 7 pinned-pinned beams coupled rigidly in chain as shown figure 8. At each junction, simple supports are introduced in order to simplify subsystems modes calculations. This produces also very large overall attenuation that demonstrate clearly the difference of predictions between SEA and SmEdA. 
In the following, rain on the roof excitation is applied on beam 1 and each beam has a damping loss factor of $0.1 \%(\eta=0.001)$, that is to say modal overlap factor less than unity (geometric mean modal overlap factor, $\bar{M}=0.0125$ ).

SmEdA model has been built for this structure decomposed in 7 subsystems. The boundary conditions used to extract the uncoupled subsystems modes are clamped - clamped for beams 2, 4 and 6 (blocked subsystems), and pinned-pinned for beams 1, 3, 5 and 7 (free subsystems).

Figure 9 presents modal energies distributions in beams. The main tendency that appears is an increase of beam modal energies disparities, subsystem after subsystem. Then, equipartition assumption lead to gross overestimates of energy transmission far from the excited beam (see also [7]).

The disparities come from resonance frequency coincidences that render one modal interaction dominant. Of course, the probability of having the phenomena increases with the number of coupled subsystems. In the case of high modal overlap, this effect has no more a significant role and as shown figure 10 equipartition of modal energy is achieved.

\subsection{HETEROGENEOUS SUBSYSTEMS}

Classical SEA supposes a vibratory diffuse wave field in each subsystem ([19]), then, SEA substructuring should be achieved to respect this requirement. However, for industrial structures, like panel stiffened by spars and stringers, it is not possible to have a diffuse field because of heterogeneity. The assumption of diffuse field of the wave approach can be related to the equipartition assumption of the modal approach. Because SmEdA is not assuming equipartition, one can expect it can be used for heterogeneous structures. To make clear this point, let us study four Euler-Bernoulli beams coupled rigidly at each end with an intermediary support (see figure 11). The external ends are simply supported for beam 1 and clamped for beam 4 . Beam 1 is excited by rain on the roof excitation.

The substructuring which is considered is shown figure 11: beam 1 and beam 4 are independant subsystems (subsystem 1 and 3, respectively), whereas beam 2 and 3 constitute a single non homogeneous subsystem (subsystem 2). 
Although analytic modal extraction can be performed, finite elements method was used to calculate the modal information of each subsystem. Modal coupling loss factors are obtained by the technique used in [1] to calculate coupling loss factors from FEM data. The modal information for each mode is eigenfrequency, generalised mass and mode shape at the coupling ends in terms of nodal displacement for free junctions and of nodal force for blocked junctions. Expression (80) of reference [1] is used to calculate interaction modal works, and then, modal coupling loss factors with (13).

For the octave band of central frequency $1000 \mathrm{hz}$, the energy ratio between the receiving beam 4 and the excited beam 1 is equal to $-36.3 \mathrm{~dB}$ using SmEdA and $-24.0 \mathrm{~dB}$ using SEA whereas a reference energy ratio obtained by FE numerical experiments (see [31]) is $-38.3 \mathrm{~dB}$. An error of $14.3 \mathrm{~dB}$ is then made by SEA whereas SmEdA gives a accurate prediction. This can be explained again by observing the modal energies distribution of subsystem 2 (figure 12) where important variations can be noticed on the modal distribution. SEA being not able to describe these variations, it overestimates the energy transfer between subsystems 2 and 3. The heterogeneity of subsystem 2 produces local modes as illustrated figure 13. The mode shapes act on the modal coupling loss factors via the interaction modal works. So, the difference of mode shape amplitudes at each end of the subsystem implies the mode is lightly coupled with modes of the right subsystem and strongly coupled with the ones of left subsystem (or vice versa). This introduces a strong disparity of mode energies which can only be taken into account by considering each mode independently. This explains why SEA fails and SmEdA gives good results. In this specific demonstration-case, it can be argued, however, that intuitively, one would actually choose four SEA elements for the analysis, and such a choice would give similar good agreement for beam 4 , namely $-34.3 \mathrm{~dB}$.

\subsection{CASE OF LOCALISED EXCITATION}

In section 4.1. and 4.2., the external sources were of rain on the roof type. This excitation is considered in SEA because it produces decorrelation of generalized forces and thus respect of modal energies equipartition for the excited subsystem (see [23]). However, in practical situations, sources cannot always be assimilated to rain on the roof excitation, leading to difficulty in using SEA, contrary to SmEdA. 
Let us study two thin steel plates coupled in an L shape. Plate 1 is excited by one white noise point force. Each plate is simply supported on non coupled edges and flexural motions are considered (see figure 14).

Let us assume plate 2 is thinner than plate 1 . Then, according to DMF, plate 1 is described by free modes at junction and plate 2 by blocked modes. These modal information are given in appendix A. The interaction modal work between mode $(m, n)$ of plate 1 and mode $(r, s)$ of plate 2 writes:

$$
\left\{\begin{array}{l}
\left.\mathbf{W}_{m n, r s}=\frac{E_{2} b h_{2}{ }^{3} \kappa_{r}}{12\left(1-v_{2}{ }^{2}\right.}\right)\left(\frac{m \pi}{a_{1}}\right)\left(\frac{s \pi}{b}\right)^{2} \sin \left(\frac{s \pi}{b} \sqrt{\kappa_{r}+1}\right), \text { if } n=s, \\
\mathbf{W}_{m n, r s}=0, \text { if } n \neq s,
\end{array}\right.
$$

where:

- $a \alpha, b, h_{\alpha}, E_{\alpha}, v_{\alpha}$ are, respectively, longitudinal length, length of the common edge, thickness, Young modulus, and Poisson's ratio for plate $\alpha, \alpha=1,2$, and;

$-\kappa_{r}$ is a modal parameter given in appendix A.

It can be noticed that the interaction modal work (26) is zero for couple of modes which have not the same index on the common edge $(n \neq s)$.

The power injected by the driving force located at point $\left(x_{i}, y_{i}\right)$ in mode $(m, n)$ of plate 1 writes:

$$
P_{i n j}^{m n}=\frac{\pi S_{f}}{\rho_{1} h_{1} a_{1} b}\left[\sin \left(\frac{m \pi}{a_{1}} x_{i}\right) \sin \left(\frac{n \pi}{b} y_{i}\right)\right]^{2},
$$

where $S_{f}$ is the power spectral density of force for the frequency band of interest $\left(\mathrm{N}^{2} /(\mathrm{rad} / \mathrm{s})\right)$, and, $\rho_{1}$ is mass density of plate 1 .

The modal injected power can vary strongly from one mode to another, contrary to the case of rain on the roof excitation.

SmEdA application was made using (27) to evaluate modal input power. Twelve different excitation positions were studied; theirs coordinates are given table 2. Figure 15 shows plates energies ratio, for excitation in the third octave band of central frequency $1000 \mathrm{~Hz}$. A good agreement can be noticed between SmEdA and reference results (obtained by DMF calculation taking into account modes belonging to the octave band of central frequency $1000 \mathrm{~Hz}$ ). This is due to the variation of modal energies as it is clearly demonstrated figure 16. The variation of modal injected power 
introduced a variation of modal energy in the excited plate, that was accentuated by modal coupling filtering effect for the modal energy distribution in plate 2: in the excited band, 20 resonant modes contributes to vibrations for plate 1 and 17 for plate 2, but due to null interaction modal work only 9 modes of plate 2 are significantly excited.

\section{CONCLUSIONS}

In this paper is presented a reformulation of SEA taking modal energy distribution into account. The necessary data to built the model are modal information of each uncoupled subsystem: natural frequencies, modal masses, modal damping and mode shapes on the coupling boundaries. Finite Element Method can be used to calculate modal information in the cases of complex subsystems that permit to apply this technique to industrial structures. The results are given in terms of modal energies which can be added to calculate subsystem energies.

It has been shown on simple examples the interest of SmEdA.

The coupling of subsystems of low modal overlap factors which has been abundantly discussed in the literature can be solved by SmEdA. In this case, the modal energy distribution is non uniform due to the effect of frequency and space coincidences.

Heterogeneous subsystems energies can be predicted by SmEdA that presents a high interest when dealing with industrial structures.

Localised excitation can lead to important variations in the modal energies distribution of the excited subsystem that cannot be described by classical SEA. A coupled plates example has shown that SmEdA can correctly predict the effect of source position.

Finally, SmEdA can be easily mixed with classical SEA, in order to calculate energy distribution only in subsystems where it is necessary and use SEA for others subsystems. This constitutes a practical interest of SmEdA. To apply SEA to coupled subsystems necessitate that all subsystems verify SEA assumption. It is very restrictive for industrial practical application because some subsystems are highly heterogeneous, locally excited or have weak modal overlap. In this case, the coupling of SmEdA for these subsystems and SEA description for others permit to solve the problem. Of course, the use of SmEdA necessitate to calculate the uncoupled subsystem modes, but is only required for these particular subsystems for which SmEdA is useful. 


\section{ACKNOWLEGMENTS}

The authors are grateful for the interest and financial support of DGA/DSP Paris (Direction des Systèmes de forces et de Prospective) and DGA/CTSN Toulon (Centre Technique des Systèmes Navals), without which this work would not have been done. 


\section{REFERENCES}

[1] L. MAXIT and J.-L. GUYADER 2001 Journal of Sound and Vibration 239, 907-948. Estimation of SEA coupling loss factors using a dual formulation and FEM modal information, part $1:$ theory, part $2:$ numerical applications.

[2] L. MAXIT 2000 Ph.D. Thesis, Institut National des Sciences Appliquées de Lyon, France. Extension et reformulation du modèle SEA par la prise en compte de la répartition des énergies modales.

[3] R.H. LYON and R.G. DEJONG 1995 Theory and Application of Statistical Energy Analysis, Butterworth-Heineman.

[4] C.B. BURROUGHS, R.W. FISCHER, and F.R. KERN 1997 The Journal of the Acoustical Society of America 101, 1779-1789. An introduction to statistical energy analysis.

[5] E.E. UNGAR 1966 U.S. Air Force AFFDL-TR 66-52, April. Fundamentals of Statistical Energy Analysis of Vibrating Systems.

[6] A.J. KEAN and W.G. PRICE 1987 Journal of Sound and Vibration 117, 363-386. Statistical energy analysis of strongly coupled systems.

[7] F.F. YAP and J. WOODHOUSE 1996 Journal of Sound and Vibration 197, 351-371. Investigation of damping effects on statistical energy analysis of coupled structures.

[8] C. FREDO 1995 Ph.D. Thesis, Chalmers University of Technology, Sweden. Statistical energy analysis and the individual case.

[9] S. FINNVEDEN 1990 Internoise90, Gothenburg, Sweden, August 90. Energy flows within a three element structure with statistical description of the design parameters.

[10] B.R. MACE and P.J. SHORTER 1997 IUTAM symposium on Statistical Energy Analysis, Southampton, UK, 8-11 July. Irregularity, damping, and coupling strength in sea.

[11] B.R. MACE and J. ROSENBERG 1998 Journal of Sound and Vibration 212, 395-415. The sea of two coupled plates : an investigation into the effects of subsystem irregularity.

[12] R.S.MING, and J. PAN 1997 Fifth International Congress on Sound and Vibration, Adelaide, Australia, December. The limitation in the sea prediction of power transmission and energy distribution:

[13] W.S. PARK, D.J. THOMPSON and N.S. FERGUSON 2000 NOVEM Meeting, Lyon, France, September. Sources of error and confidence intervals for SEA parameters.

[14] F.J. FAHY, and A.D. MOHAMMED 1992 Journal of Sound and Vibration 158, 45-67. A study of uncertainty in applications of sea to coupled beam and plate systems, part I : Computational experiments. 
[15] B.R. MACE 1993 Journal of Sound and Vibration 166, 429-461. The statistical energy analysis of two continuous one-dimensional subsystems.

[16] B.R. MACE 1994 Journal of Sound and Vibration 178, 95-112. On the statistical energy analysis hypothesis of coupling power proportionality and some implications of its failure.

[17] S. FINNVEDEN 1995 Journal of Sound and Vibration 187, 495-529. Ensemble averaged vibration energy flows in a three-element structure.

[18] S. FINNVEDEN 1998 Internoise98, Christchurch, New Zealand, 16-18 November. Coupling strength criterion for the modal approach to statistical energy analysis of spring coupled elements.

[19] R.S. LANGLEY 1992 Journal of Sound and Vibration 159, 485-502. A wave intensity technique for the analysis of high frequency vibration.

[20] R.S. LANGLEY and A.N. BERCIN 1994 Philosophical Transactions Royal Society London A346, 489-499. Wave intensity analysis of high frequency vibrations.

[21] H. NISHINO and M. OHLRICH 2000 NOVEM Meeting, Lyon, France, September. Prediction of medium frequency vibration by wave intensity analysis.

[22] J.-G. IH and K.S. CHAE 2000 NOVEM Meeting, Lyon, France, September. Use of the ray tracing method for predicting the vibration energy distribution in the thin plate at high frequencies.

[23] E.K. DIMITRIADIS and A.D. PIERCE 1988 Journal of Sound and Vibration 123, 397-412. Analytical solution for the power exchange between strongly coupled plates under random excitation : a test of statistical energy analysis concepts.

[24] C. BOISSON, J.-L. GUYADER, and C. LESUEUR 1985 Acustica 58, 223-233. Étude numérique de la transmission d'énergie vibratoire entre structures assemblées: cas d'assemblages en $\mathrm{L}$, $\mathrm{T}$ et + .

[25] G. FORTUNATO, and K. DE LANGHE 1999 Sixth International Congress on Sound and Vibration, Copenhagen, Denmark, 5-8 July. The influence of boundary conditions on sea parameters in the low and high frequency range.

[26] R.S. LANGLEY 1989 Journal of Sound and Vibration 135, 499-508. A general derivation of the statistical energy analysis equations for coupled dynamic systems.

[27] H.G. DAVIES 1973 The Journal of the Acoustical Society of America 54, 507-515. Random vibration of distributed systems strongly coupled at discrete points.

[28] G. MAIDANIK and J. DICKEY 1987 The winter annual meeting of the American society of mechanical engineers, Massachusetts USA, 13-18 December. Modal and wave approaches to the Statistical Energy Analysis (SEA). 
[29] B.R. MACE 1997 Fifth International Congress on Sound and Vibration, Adelaide, Australia, December. Energy flow and s.e.a. at low modal overlap.

[30] T.D. SCHARTON and R.H. LYON 1968 The Journal of the Acoustical Society of America 43, 1332-1343. Power flow and energy sharing in random vibration.

[31] L. MAXIT and J.-L. GUYADER 2000 NOVEM Meeting, Lyon, France, September. Structural vibration analysis of the CLIO II firewall using SEA model and CLF-DMF technique.

[32] J.-L. GUYADER 1999 Sixth International Congress on Sound and Vibration, Copenhagen, Denmark, 5-8 July. State of the art of energy methods used for vibro acoustic predictions.

[33] W. SOEDEL 1993 Vibrations of shells and plates. Second edition. - New York : Marcel Dekker, Inc. 
The structure and the co-ordinate systems are shown figure 14. According with the DMF, plate 1 is described by modes of the uncoupled - free subsystem and plate 2 by modes of the uncoupled blocked subsystem. Thus, the modal information of plate 1 are obtained by considering the plate simply supported on its four edges :

$$
\left\{\begin{array}{l}
\bullet \omega_{m n}=\sqrt{\frac{h_{1}^{2} E_{1}}{12\left(1-v_{1}^{2}\right) \rho_{1}}\left(\left(\frac{m \pi}{a_{1}}\right)^{2}+\left(\frac{n \pi}{b}\right)^{2}\right),} \\
\bullet M_{m n}=\frac{\rho_{1} h_{1} a_{1} b_{1}}{4}, \\
\bullet \tilde{W}^{m n}\left(x_{1}, y_{1}\right)=\sin \left(\frac{m \pi}{a_{2}} x_{1}\right) \sin \left(\frac{n \pi}{b} y_{1}\right),
\end{array}\right.
$$

and, those of plate 2 by considering the plate clamped on the coupling edge $x_{2}=0$ and simply supported on the others edges (see the technique of calculation in [33], p. 84-85) :

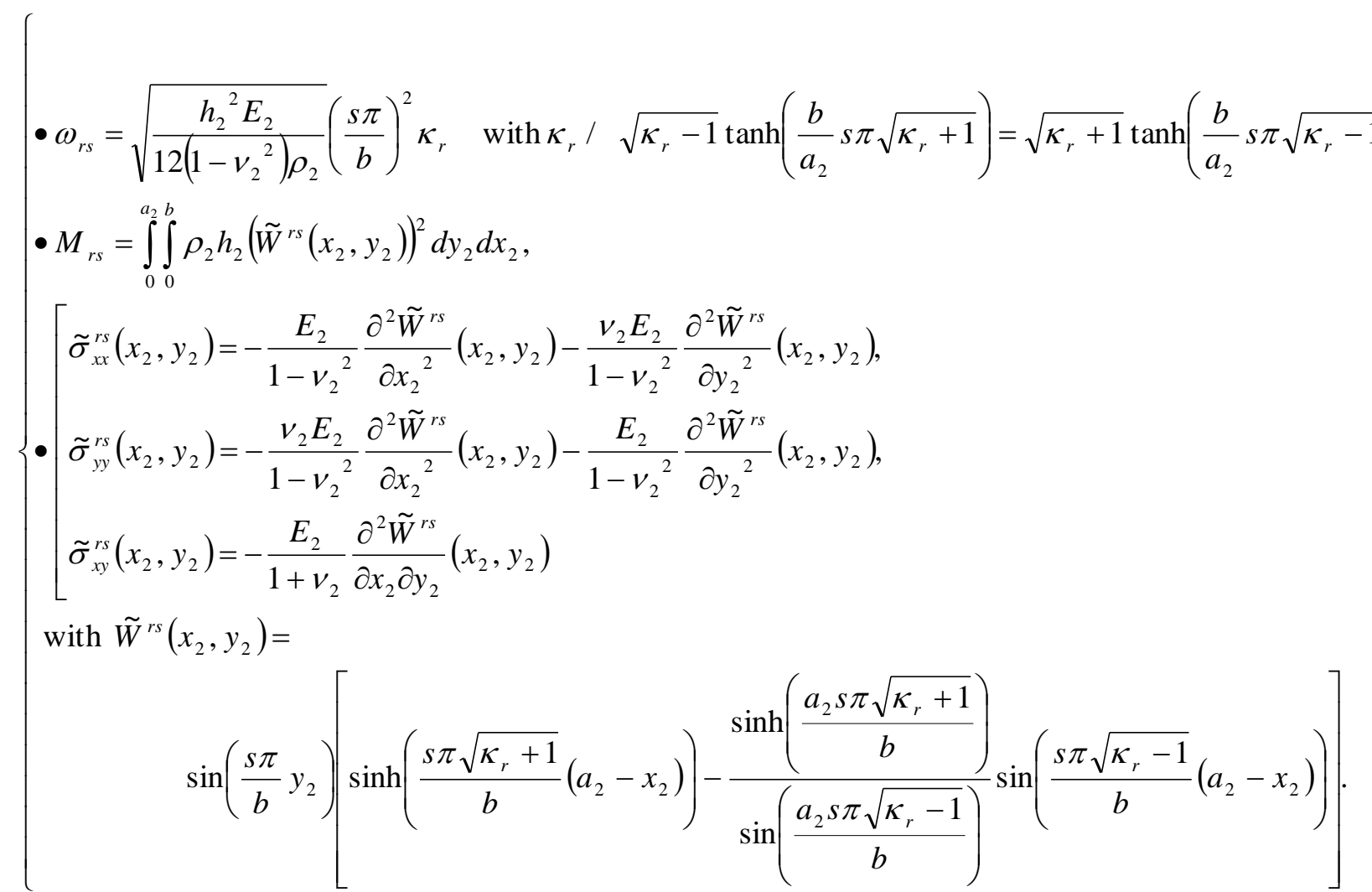

where $\tilde{W}^{m n}$ is the displacement mode shapes of plate 1 and $\tilde{\sigma}_{x x}^{r s}, \tilde{\sigma}_{y y}^{r s}, \tilde{\sigma}_{x y}^{r s}$ are the stress mode shapes of plate 2 . 
For the case of two plates coupled in an L-shape, the interaction modal work between the mode $(m, n)$ of plate 1 and the modes $(r, s)$ of plate 2 is given by :

$$
\mathbf{W}_{m n, r s}=\frac{b h_{2}^{3}}{12} \int_{0}^{b} \frac{\partial \tilde{W}^{m n}}{\partial x_{1}}\left(a_{1}, y\right) \tilde{\sigma}_{x x}^{r s}(0, y) d y,
$$

and can be rewritten by using the mode shapes (A3), (A6) :

$$
\left\{\begin{array}{l}
\left.\mathbf{W}_{m n, r s}=\frac{E_{2} b h_{2}{ }^{3} \kappa_{r}}{12\left(1-v_{2}{ }^{2}\right.}\right)\left(\frac{m \pi}{a_{1}}\right)\left(\frac{s \pi}{b}\right)^{2} \sin \left(\frac{s \pi}{b} \sqrt{\kappa_{r}+1}\right), \text { if } n=s, \\
\mathbf{W}_{m n, r s}=0, \text { if } n \neq s,
\end{array}\right.
$$




\section{FIGURE CAPTIONS}

Figure 1. Coupling of two continuum mechanical systems.

Figure 2. Representation of the uncoupled subsystems. (a) Uncoupled-free subsystem 1 ; (b) Uncoupled-blocked subsystem 2.

Figure 3. Illustration of the interaction between $N_{1}$ modes of subsystem 1 and $N_{2}$ modes of subsystem 2 .

Figure 4. Illustration of the rigid coupled beams.

Figure 5. Beam energy ratio, $E_{2} / E_{1}$, versus the geometric mean of the modal overlap factors, $\sqrt{M_{1} M_{2}}$ (octave band $1000 \mathrm{hz}$ ). Comparison of four calculations : x, exact ; - - SmEdA ; ----, SEA ${ }^{\text {DMF }}$; *, SEA ${ }^{\text {wave }}$. $\mathrm{L}_{1}=2.4 \mathrm{~m}, \mathrm{~L}_{2}=1.2 \mathrm{~m}, \mathrm{~b}_{1}=\mathrm{b}_{2}=0,01 \mathrm{~m}, \mathrm{~h}_{1}=3 \mathrm{~mm}, \mathrm{~h}_{2}=1 \mathrm{~mm}, \mathrm{E}_{1}=\mathrm{E}_{2}=7.10^{10} \mathrm{~Pa}$, $\rho_{1}=\rho_{2}=2700 \mathrm{~kg} / \mathrm{m}^{3} . \mathrm{N}_{1}=10$ modes, $\mathrm{N}_{2}=9$ modes.

Figure 6. Modal energy distribution of the receiving beam ( $\mathrm{dB}$, ref. $10^{-12}$ joule). Resonant modes classified with increasing natural frequencies. Four cases: (a) $\bar{M}=7,9.10^{-3}$; (b) $\bar{M}=0.09$; (c) $\bar{M}=0.18$; (d) $\bar{M}=1.43$. Two calculations : -o-o-, SmEdA ; -+--+-, $\mathrm{SEA}^{\mathrm{DMF}}$.

Figure 7. Modal coupling loss factors, $\eta_{p q}$. (Modes classified with increasing natural frequencies.) Same cases than the previous figure : (a) $\bar{M}=7,9.10^{-3}$; (b) $\bar{M}=0.09$; (c) $\bar{M}=0.18$; (d) $\bar{M}=1.43$.

Figure 8. Schematic representation of the 7 rigidly coupled beams. Rain on the roof excitation on beam 1.

$\mathrm{L}_{1}=2,4 \mathrm{~m}, \mathrm{~L}_{2}=1,2 \mathrm{~m}, \mathrm{~L}_{3}=2,2 \mathrm{~m}, \mathrm{~L}_{4}=1,4 \mathrm{~m}, \mathrm{~L}_{5}=2 \mathrm{~m}, \mathrm{~L}_{6}=1,2 \mathrm{~m}, \mathrm{~L}_{7}=2,3 \mathrm{~m}, \mathrm{~h}_{1}=3 \mathrm{~mm}, \mathrm{~h}_{2}=1 \mathrm{~mm}, \mathrm{~h}_{3}=4$ $\mathrm{mm}, \mathrm{h}_{4}=1,2 \mathrm{~mm}, \mathrm{~h}_{5}=2 \mathrm{~mm}, \mathrm{~h}_{6}=0,8 \mathrm{~mm}, \mathrm{~h}_{7}=3,5 \mathrm{~mm}, \mathrm{~b}_{\alpha}=0,01 \mathrm{~m}, \mathrm{E}_{\alpha}=7.10^{10} \mathrm{~Pa}, \rho_{\alpha}=2700 \mathrm{~kg} / \mathrm{m}^{3}$, $\eta_{\alpha}=0.001(\alpha \in[1,7])$. 
Figure 9. Modal energy distributions of the 7 beams in the case of weak modal overlap: $\eta_{\alpha}=0.001$ $(\alpha \in[1,7])$. Octave band of central frequency $1000 \mathrm{~Hz}$. Two calculations: -o-o-, SmEdA; -+-+-, SEA $^{\mathrm{DMF}}$.

Figure 10. Modal energy distributions of the 7 beams in the case of geometric mean modal overlap factors equal to one (octave band of central frequency $1000 \mathrm{~Hz}$ ). Two calculations : -o-o-, SmEdA ; -+-+-, SEA ${ }^{\text {DMF. }}$

Figure 11. (a) Illustration of the 4 coupled beams; (b) Substructuring.

Beams' characteristics: $\mathrm{L}_{1}=2,4 \mathrm{~m}, \mathrm{~L}_{2}=1,2 \mathrm{~m}, \mathrm{~L}_{3}=2,2 \mathrm{~m}, \mathrm{~L}_{4}=1,4 \mathrm{~m}, \mathrm{~h}_{1}=3 \mathrm{~mm}, \mathrm{~h}_{2}=1 \mathrm{~mm}, \mathrm{~h}_{3}=5 \mathrm{~mm}$, $\mathrm{h}_{4}=1,2 \mathrm{~mm}, \mathrm{~b}_{\alpha}=0,01 \mathrm{~m}, \mathrm{E}_{\alpha}=7.10^{10} \mathrm{~Pa}, \rho_{\alpha}=2700 \mathrm{~kg} / \mathrm{m}^{3}, \eta_{\alpha}=0.01(\alpha \in[1,4])$.

Figure 12. Modal energy distribution of subsystem 2 (dB, ref. $10^{-12}$ joule). Modes classified with increasing natural frequencies. SmEdA results.

Figure 13. Examples of displacement mode shapes for subsystem 2. (a) mode 1 of the previous figure; (b) mode 2 of the previous figure.

Figure 14. Two plates coupled in an L-shape and excited by a point force $F_{i}$ on plate $1 . \mathrm{a}_{1}=1,7 \mathrm{~m}$, $\mathrm{a}_{2}=0,8 \mathrm{~m}, \mathrm{~b}=1 \mathrm{~m}, \mathrm{~h}_{1}=6 \mathrm{~mm}, \mathrm{~h}_{2}=3 \mathrm{~mm}, \mathrm{E}_{1}=\mathrm{E}_{2}=2.10^{11} \mathrm{~Pa}, \rho_{1}=\rho_{2}=7800 \mathrm{~kg} / \mathrm{m}^{3}, \eta_{1}=\eta_{2}=0,01$.

Figure 15. Total energy ratio for each excitation point. Third octave band of central frequency 1000 hz. Comparison of three calculations : o, reference obtained from DMF with non resonant modes ; ,- SmEdA ; ----, SEA.

Figure 16. Modal energy distributions for excitation case number 7 (dB, ref. $10^{-12}$ joule). (a) Plate 1. (b) Plate 2. Modes classified with increasing natural frequencies.

Table 1. $\bar{M}$, geometric mean modal overlap factor ; $\eta$, damping loss factor ; $\gamma$, gamma factor. Values for different cases of figure 5.

Table 2. $\left(x_{i}, y_{i}\right)$ co-ordinates of point excitation $i$. 


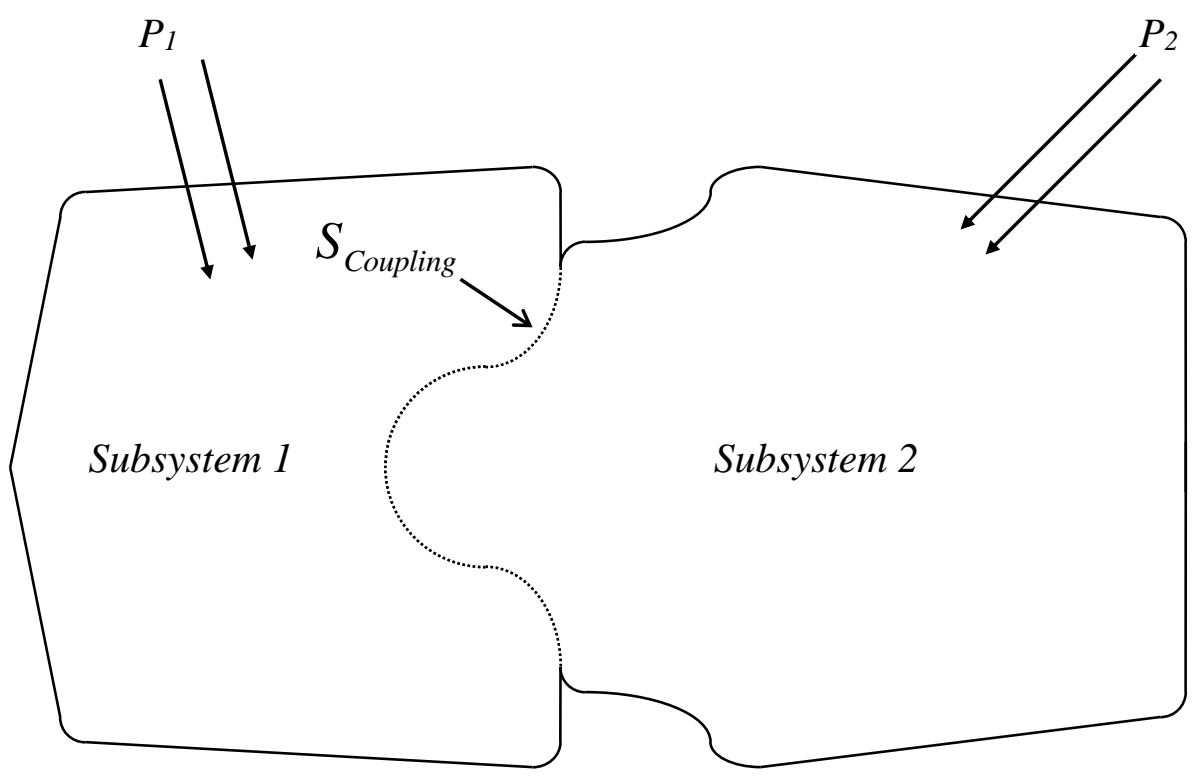

Figure 1. Coupling of two continuum mechanical systems. 


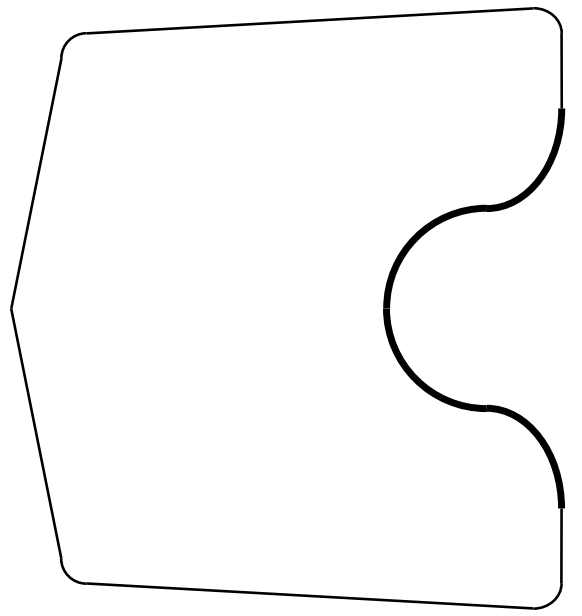

(a)

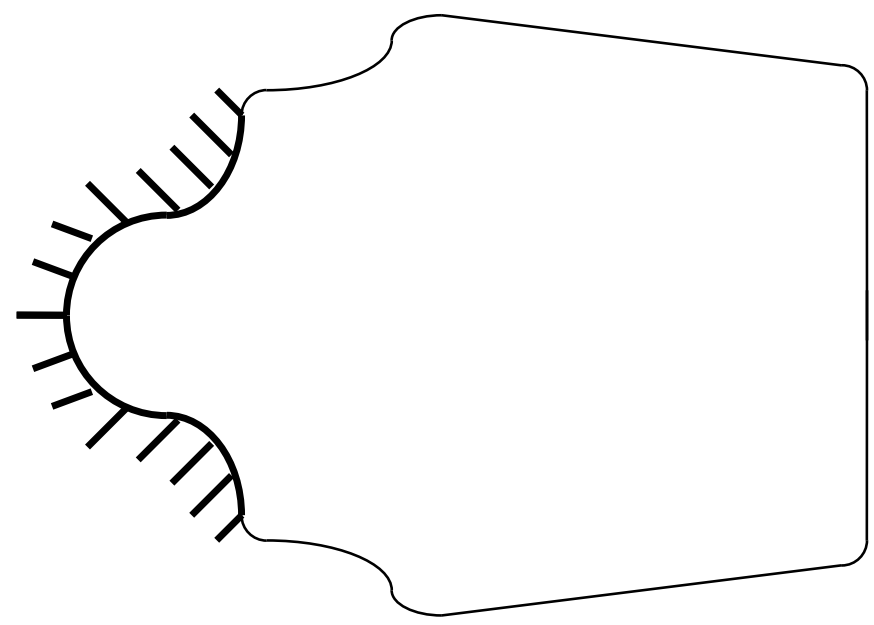

(b)

Figure 2. Representation of the uncoupled subsystems. (a) Uncoupled-free subsystem 1 ; (b) Uncoupled-blocked subsystem 2. 
FIGURE 3

L. MAXIT, J.-L. GUYADER

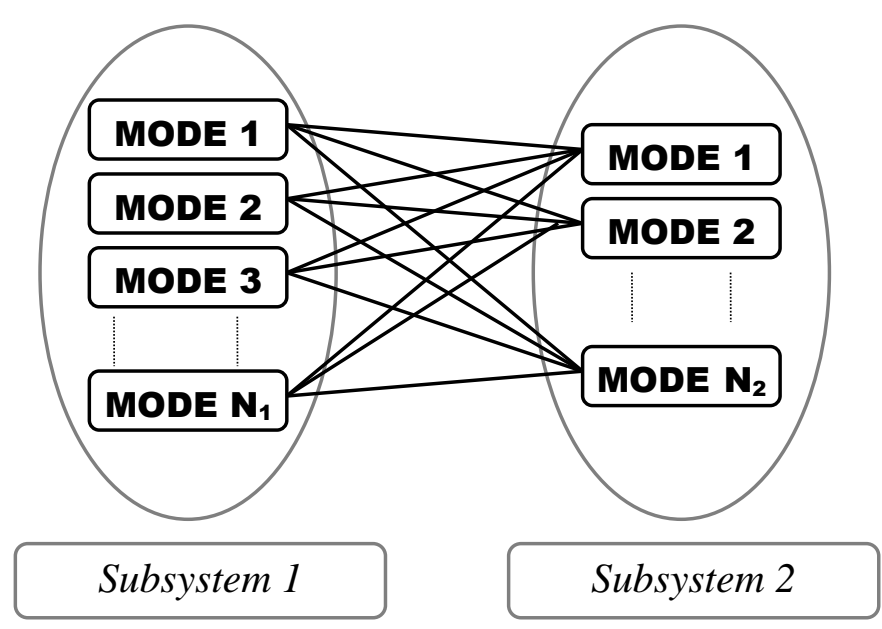

Figure 3. Illustration of the interaction between $N_{1}$ modes of subsystem 1 and $N_{2}$ modes of subsystem 2 . 


$\Delta$ Beam 1 $\triangle$

Figure 4. Illustration of the rigid coupled beams. 


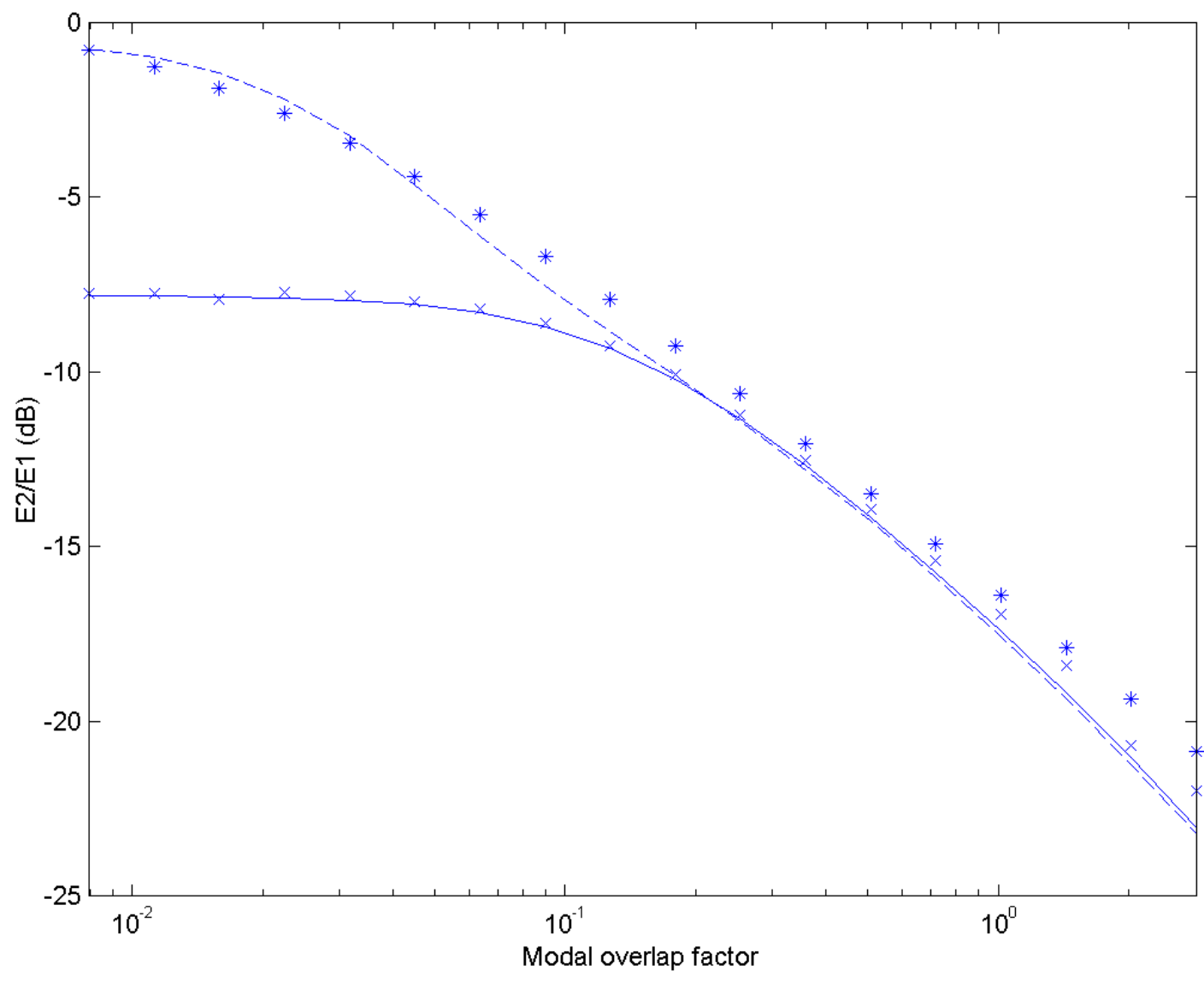

Figure 5. Beam energy ratio, $E_{2} / E_{1}$, versus the geometric mean of the modal overlap factors, $\sqrt{M_{1} M_{2}}$ (octave band of central frequency $1000 \mathrm{hz}$ ). Comparison of four calculations : x, exact ; - $\mathrm{SmEdA} ;----, \mathrm{SEA}^{\mathrm{DMF}} ;{ }^{*}, \mathrm{SEA}^{\text {wave }} \mathrm{L}_{1}=2.4 \mathrm{~m}, \mathrm{~L}_{2}=1.2 \mathrm{~m}, \mathrm{~b}_{1}=\mathrm{b}_{2}=0,01 \mathrm{~m}, \mathrm{~h}_{1}=3 \mathrm{~mm}, \mathrm{~h}_{2}=1 \mathrm{~mm}$, $\mathrm{E}_{1}=\mathrm{E}_{2}=7.10^{10} \mathrm{~Pa}, \rho_{1}=\rho_{2}=2700 \mathrm{~kg} / \mathrm{m}^{3} . \mathrm{N}_{1}=10$ modes, $\mathrm{N}_{2}=9$ modes. 
(a)
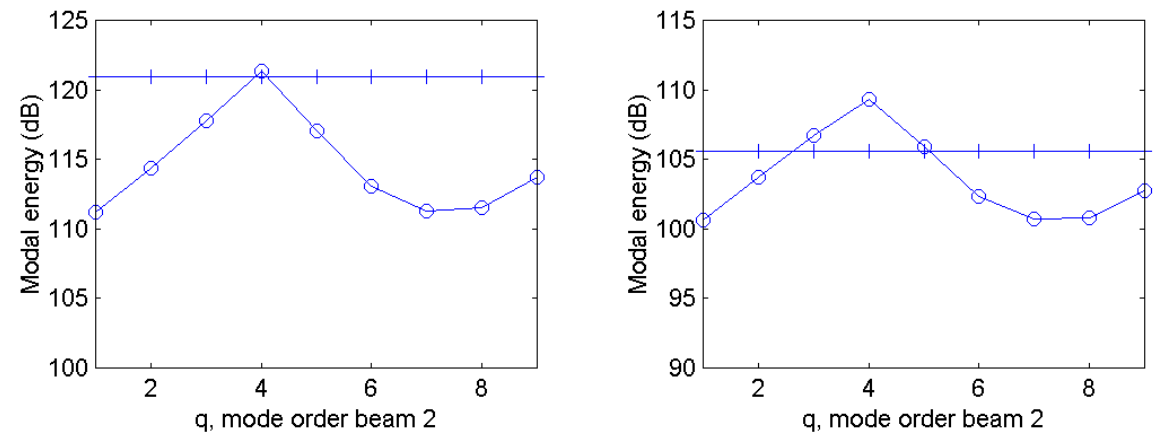

(b)
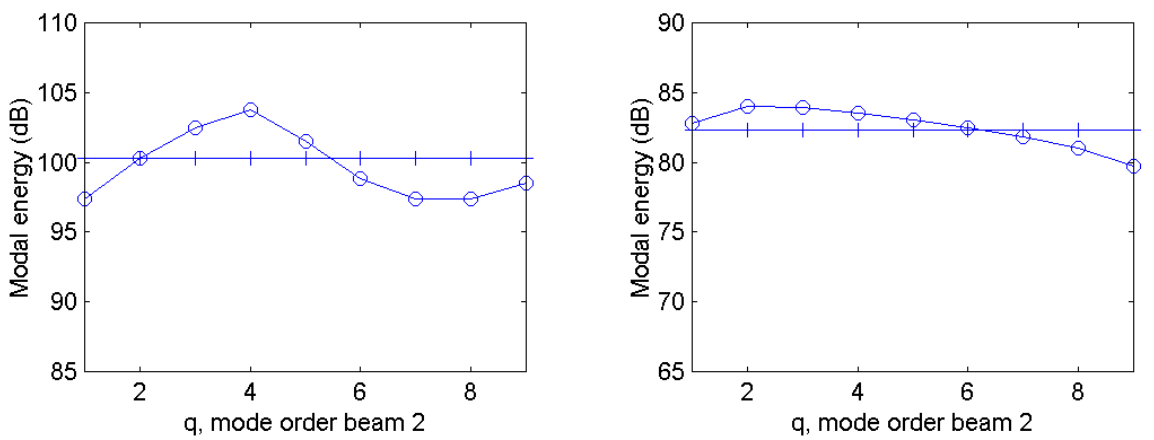

(d)

Figure 6. Modal energy distribution of the receiving beam ( $\mathrm{dB}$, ref. $10^{-12}$ joule). Resonant modes classified with increasing natural frequencies. Four cases: (a) $\bar{M}=7,9.10^{-3}$; (b) $\bar{M}=0.09$; (c) $\bar{M}=0.18$; (d) $\bar{M}=1.43$. Two calculations : $-0-0-$, SmEdA ; -+---', $\mathrm{SEA}^{\mathrm{DMF}}$. 
(a)
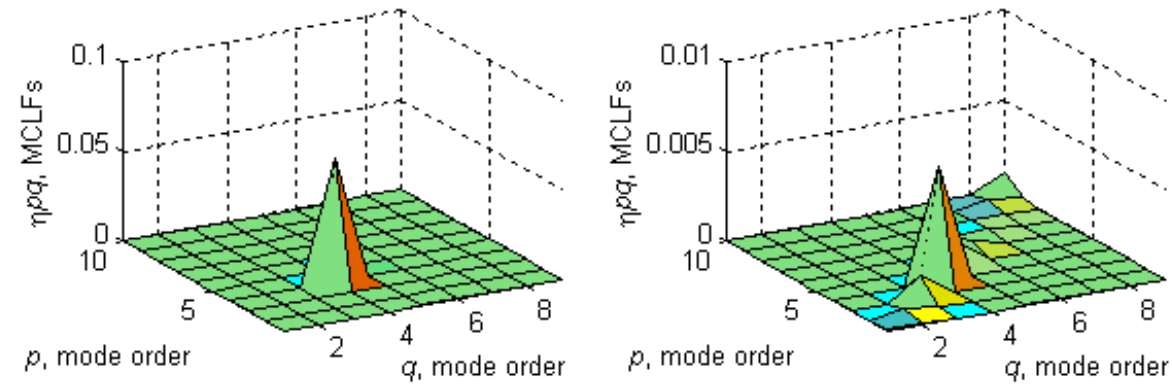

(b)

(c)
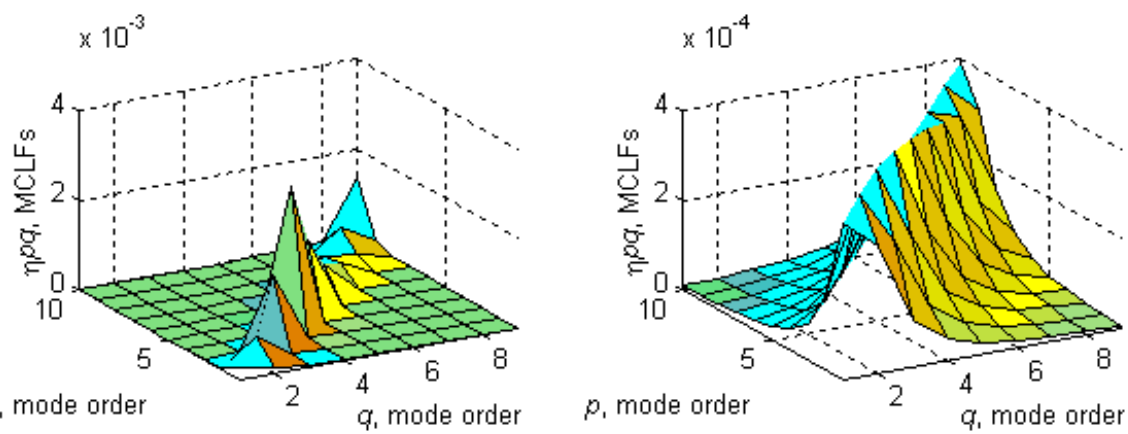

(d)

Figure 7. Modal coupling loss factors, $\eta_{p q}$. (Modes classified with increasing natural frequencies.) Same cases than the previous figure : (a) $\bar{M}=7,9.10^{-3}$; (b) $\bar{M}=0.09$; (c) $\bar{M}=0.18$; (d) $\bar{M}=1.43$. 


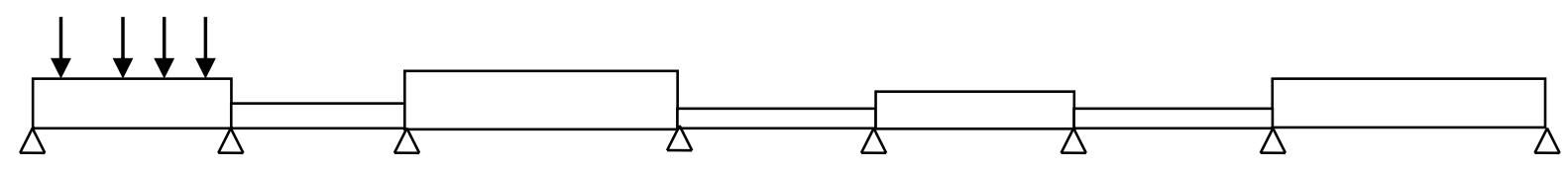

Figure 8. Schematic representation of the 7 rigidly coupled beams. Rain on the roof excitation on beam 1.

$\mathrm{L}_{1}=2,4 \mathrm{~m}, \mathrm{~L}_{2}=1,2 \mathrm{~m}, \mathrm{~L}_{3}=2,2 \mathrm{~m}, \mathrm{~L}_{4}=1,4 \mathrm{~m}, \mathrm{~L}_{5}=2 \mathrm{~m}, \mathrm{~L}_{6}=1,2 \mathrm{~m}, \mathrm{~L}_{7}=2,3 \mathrm{~m}, \mathrm{~h}_{1}=3 \mathrm{~mm}, \mathrm{~h}_{2}=1 \mathrm{~mm}, \mathrm{~h}_{3}=4$ $\mathrm{mm}, \mathrm{h}_{4}=1,2 \mathrm{~mm}, \mathrm{~h}_{5}=2 \mathrm{~mm}, \mathrm{~h}_{6}=0,8 \mathrm{~mm}, \mathrm{~h}_{7}=3,5 \mathrm{~mm}, \mathrm{~b}_{\alpha}=0,01 \mathrm{~m}, \mathrm{E}_{\alpha}=7.10^{10} \mathrm{~Pa}, \rho_{\alpha}=2700 \mathrm{~kg} / \mathrm{m}^{3}$. 


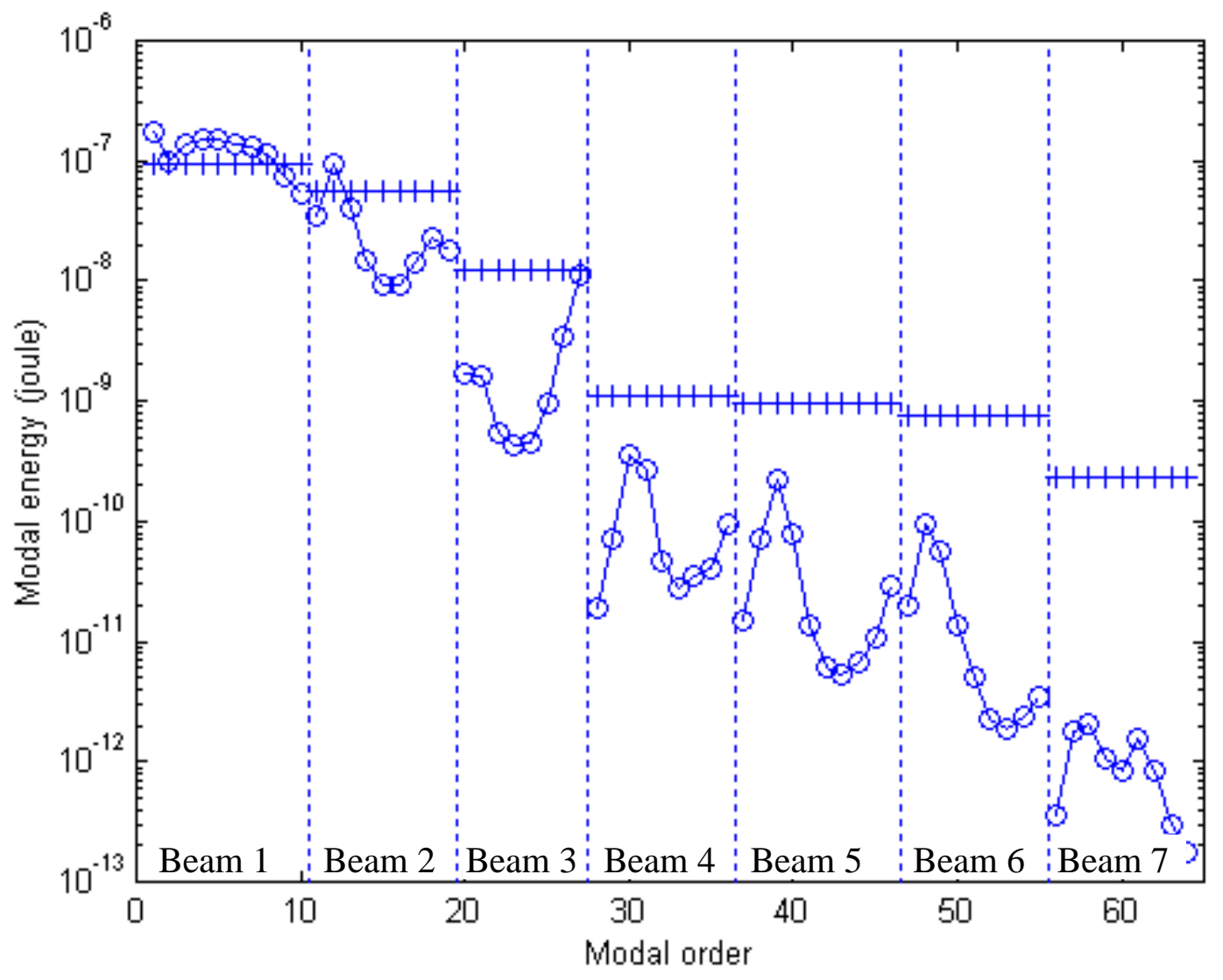

Figure 9. Modal energy distributions of the 7 beams in the case of weak modal overlap: $\eta_{\alpha}=0.001$, $\alpha \in[1,7]$. Octave band of central frequency $1000 \mathrm{~Hz}$. Two calculations:-o-o-, SmEdA; -+---, SEA $^{\mathrm{DMF}}$. 


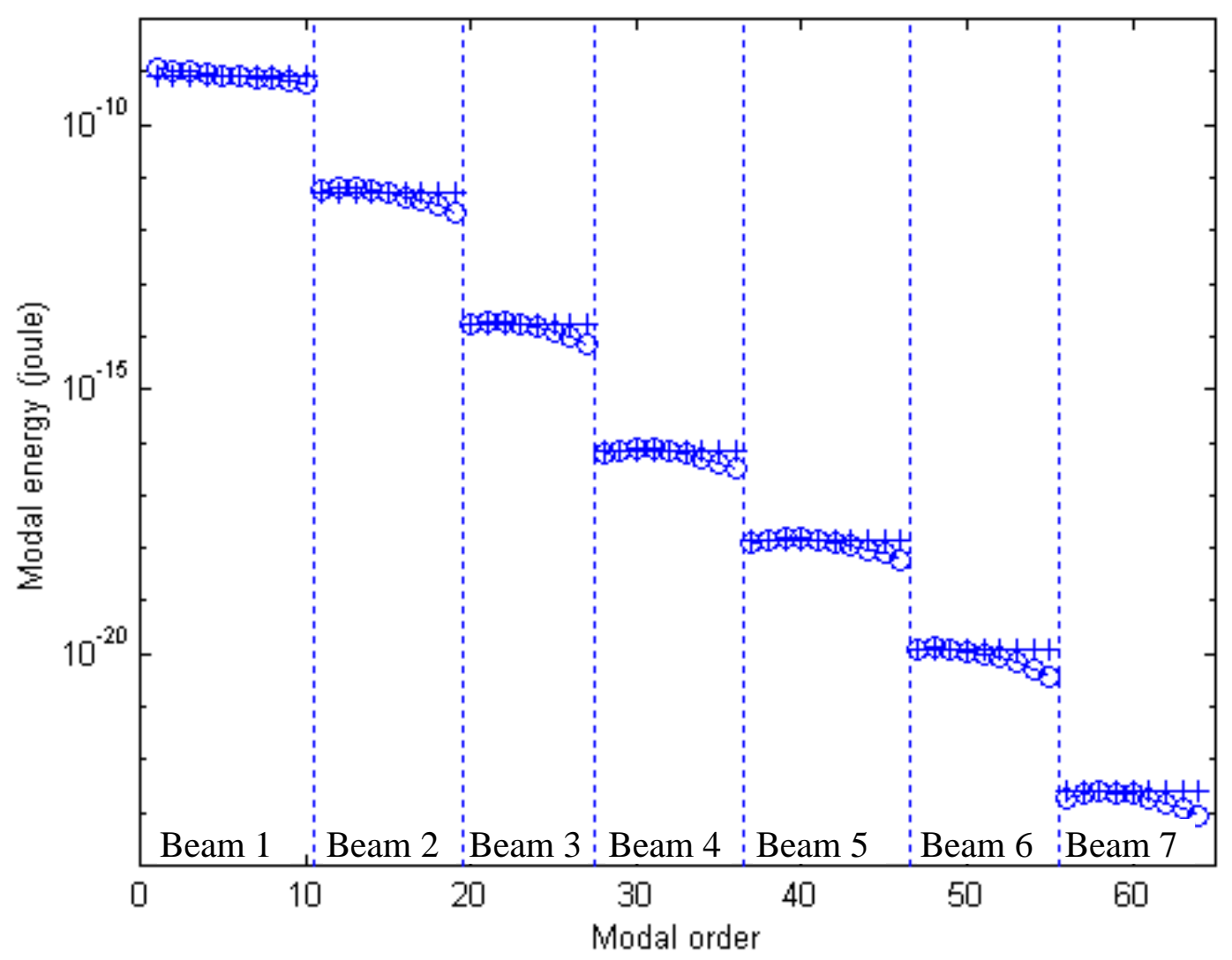

Figure 10. Modal energy distributions of the 7 beams in the case of geometric mean modal overlap factors equal to one: $\eta_{\alpha}=0.08, \alpha \in[1,7]$. Octave band of central frequency $1000 \mathrm{~Hz}$. Two calculations : -o-o-, SmEdA ; -+-+-, SEA ${ }^{\mathrm{DMF}}$. 


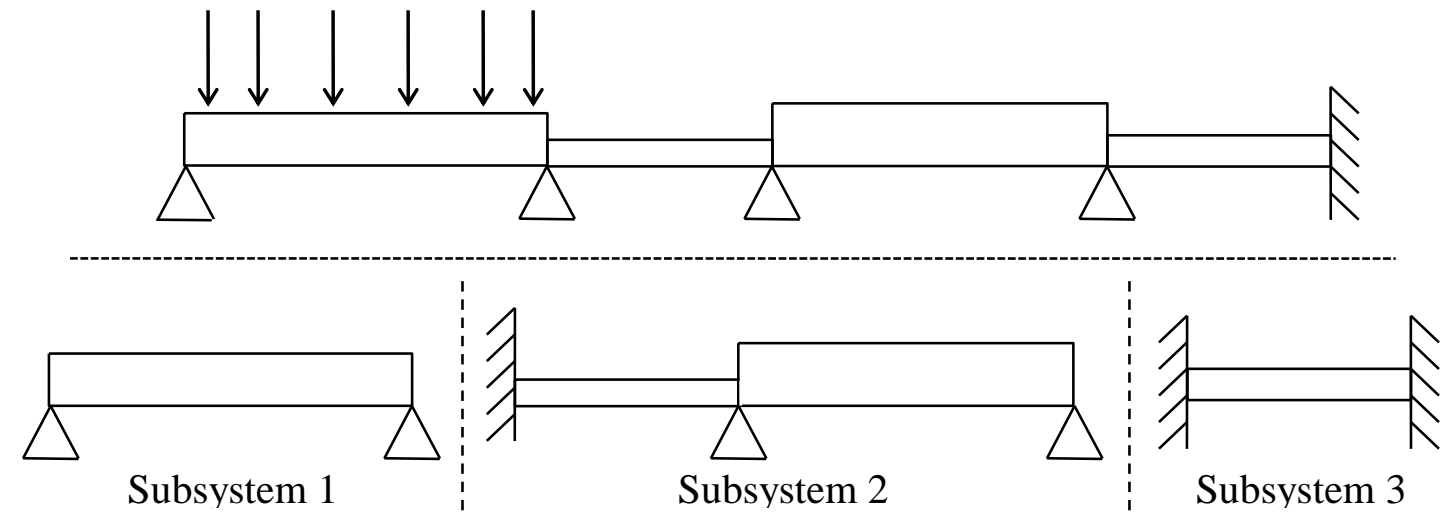

(a)

Figure 11. (a) Illustration of the 4 coupled beams; (b) Substructuring.

Beams' characteristics: $\mathrm{L}_{1}=2,4 \mathrm{~m}, \mathrm{~L}_{2}=1,2 \mathrm{~m}, \mathrm{~L}_{3}=2,2 \mathrm{~m}, \mathrm{~L}_{4}=1,4 \mathrm{~m}, \mathrm{~h}_{1}=3 \mathrm{~mm}, \mathrm{~h}_{2}=1 \mathrm{~mm}, \mathrm{~h}_{3}=5 \mathrm{~mm}$, $\mathrm{h}_{4}=1,2 \mathrm{~mm}, \mathrm{~b}_{\alpha}=0,01 \mathrm{~m}, \mathrm{E}_{\alpha}=7.10^{10} \mathrm{~Pa}, \rho_{\alpha}=2700 \mathrm{~kg} / \mathrm{m}^{3}, \eta_{\alpha}=0.01(\alpha \in[1,4])$. 


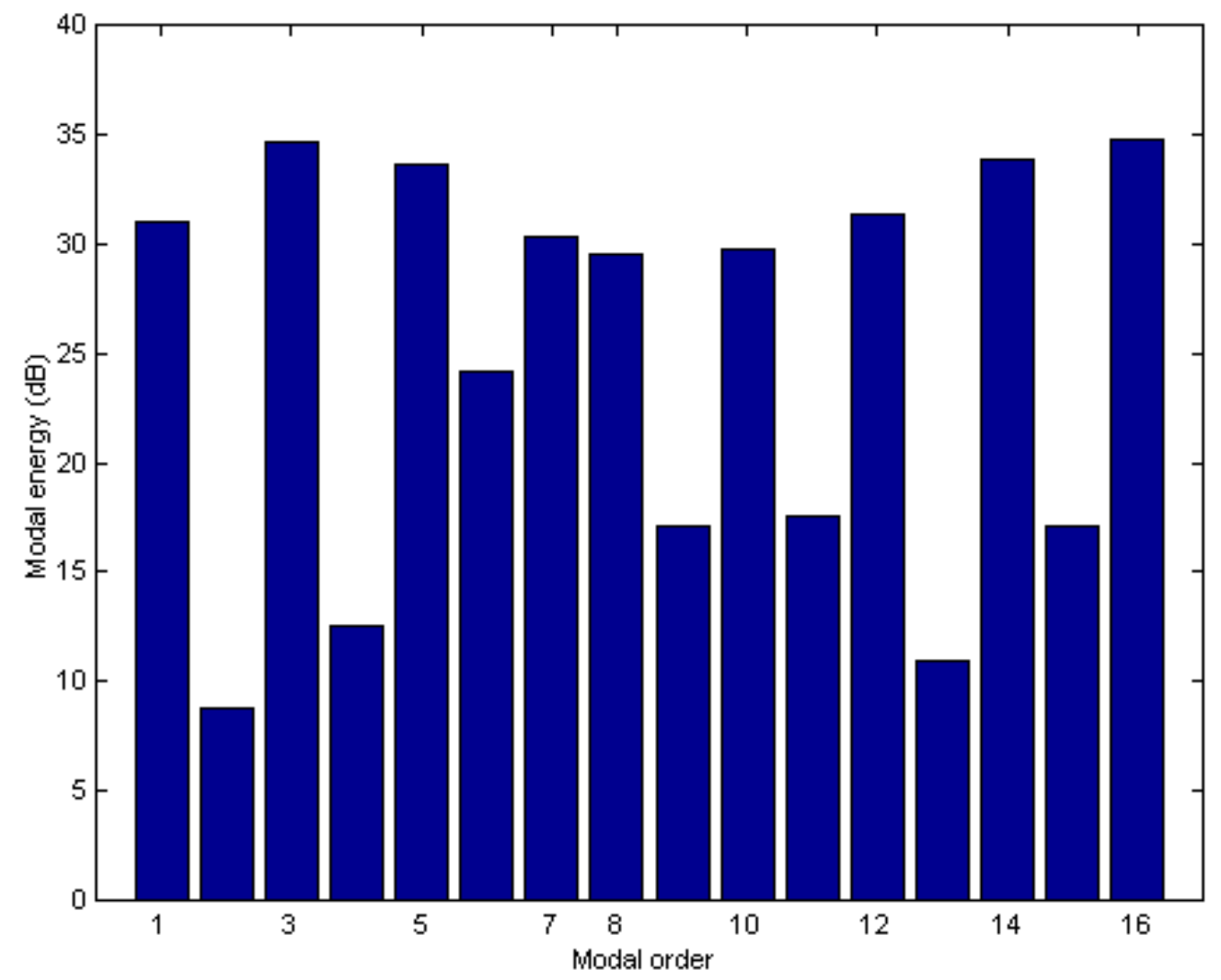

Figure 12. Modal energy distribution of subsystem 2 (dB, ref. $10^{-12}$ joule). Modes classified with increasing natural frequencies. SmEdA results. 


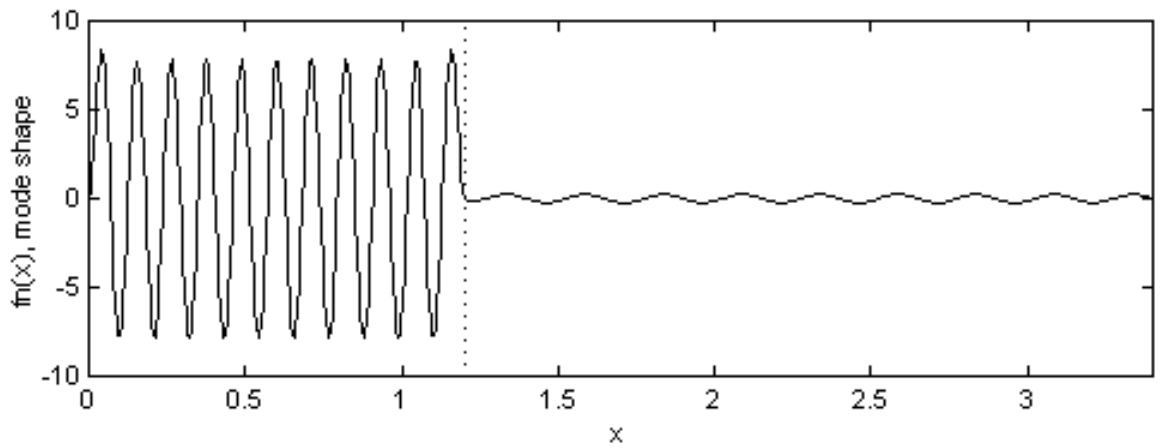

(a)

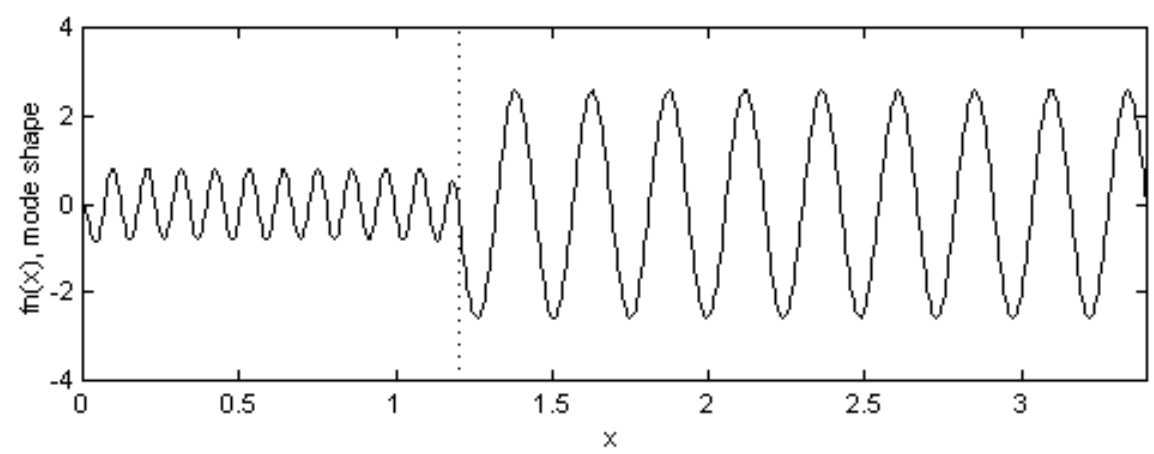

(b)

Figure 13. Examples of displacement mode shapes for subsystem 2. (a) mode 1 of the previous figure; (b) mode 2 of the previous figure. 


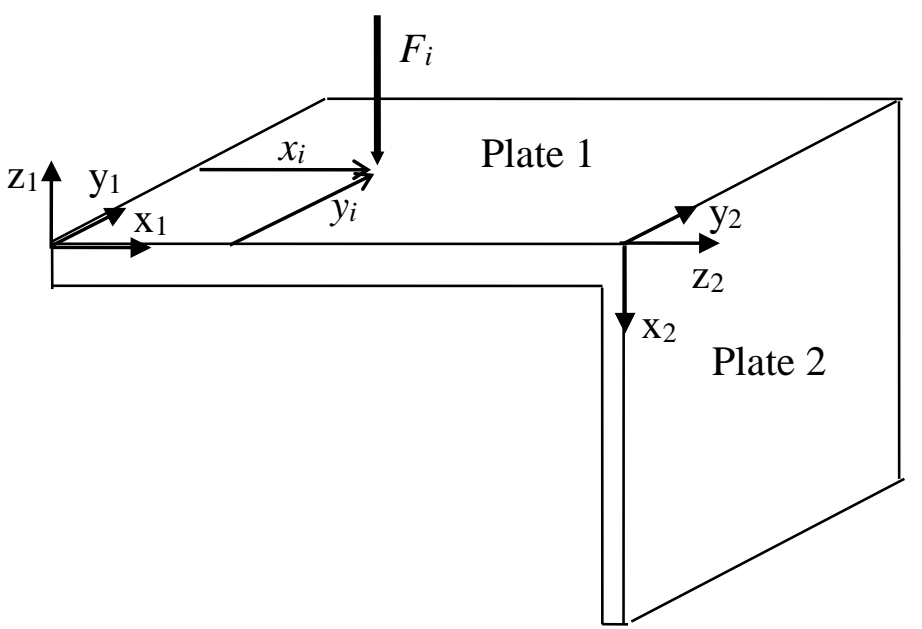

Figure 14. Two plates coupled in an L-shape and excited by a point force $F_{i}$ on plate $1 . \mathrm{a}_{1}=1,7 \mathrm{~m}$, $\mathrm{a}_{2}=0,8 \mathrm{~m}, \mathrm{~b}=1 \mathrm{~m}, \mathrm{~h}_{1}=6 \mathrm{~mm}, \mathrm{~h}_{2}=3 \mathrm{~mm}, \mathrm{E}_{1}=\mathrm{E}_{2}=2.10^{11} \mathrm{~Pa}, \rho_{1}=\rho_{2}=7800 \mathrm{~kg} / \mathrm{m}^{3}, \eta_{1}=\eta_{2}=0,01$. 


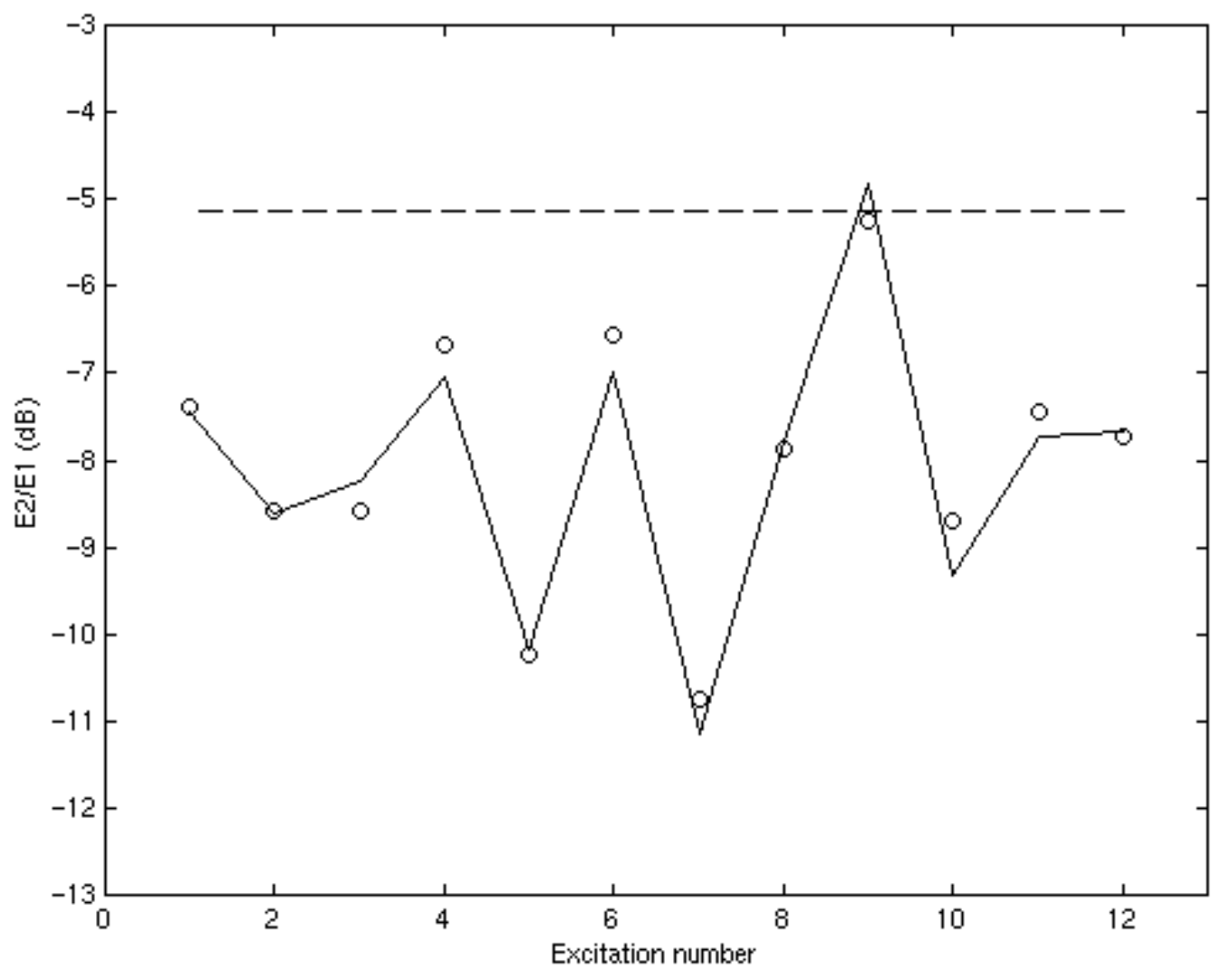

Figure 15. Total energy ratio for each excitation point. Third octave band of central frequency 1000 hz. Comparison of three calculations : o, reference obtained from DMF with non resonant modes ; -, SmEdA ; ----, SEA. 


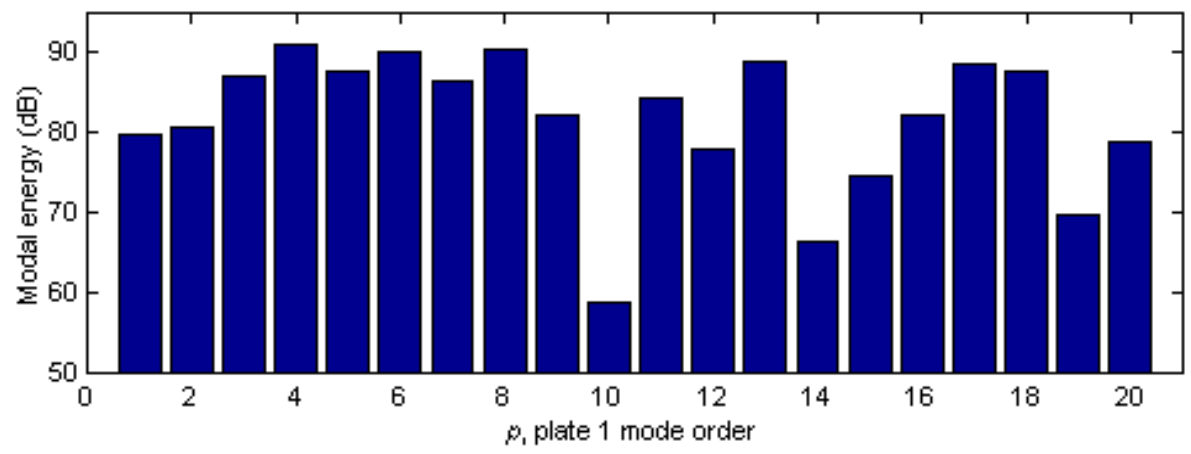

(a)

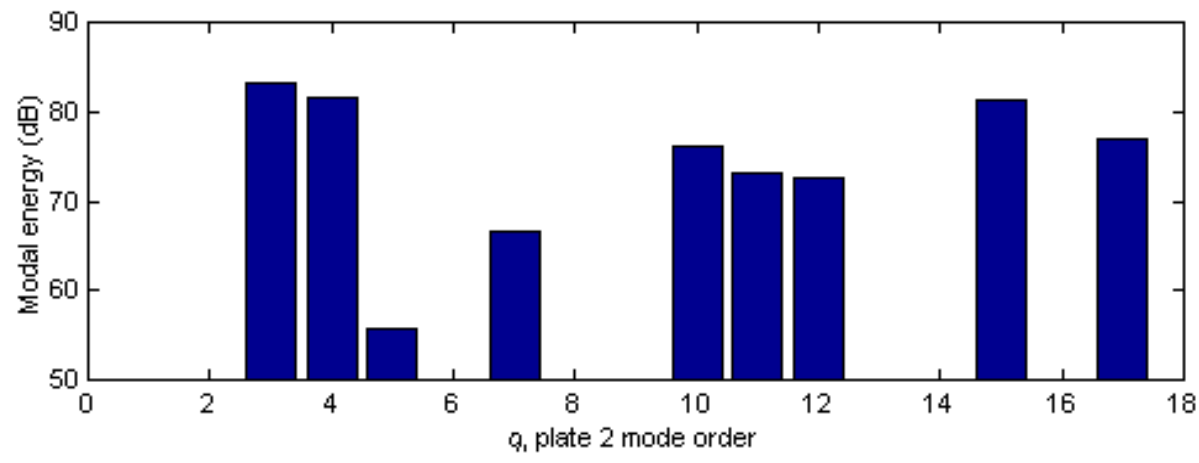

(b)

Figure 16. Modal energy distributions for excitation case number 7 (dB, ref. $10^{-12}$ joule). (a) Plate 1. (b) Plate 2. Modes classified with increasing natural frequencies. 


\begin{tabular}{c|ccccccccc}
\hline $\bar{M}$ & 0.0079 & 0.016 & 0.032 & 0.063 & 0.127 & 0.254 & 0.508 & 1.016 & 2.032 \\
\hline$\eta$ & 0.0005 & 0.001 & 0.002 & 0.004 & 0.008 & 0.016 & 0.032 & 0.064 & 0.128 \\
\hline$\gamma$ & 91.1 & 22.77 & 5.69 & 1.42 & 0.35 & 0.09 & 0.02 & 0.01 & 0.002 \\
\hline
\end{tabular}

Table 1. $\bar{M}$, geometric mean modal overlap factor $; \eta$, damping loss factor ; $\gamma$, gamma factor. Values for different cases of figure 5. 


\begin{tabular}{c|cccccccccccc}
\hline$i$ & 1 & 2 & 3 & 4 & 5 & 6 & 7 & 8 & 9 & 10 & 11 & 12 \\
\hline$x_{i}(\mathrm{~m})$ & 0.65 & 0.14 & 1.11 & 0.96 & 0.50 & 1.30 & 0.36 & 1.49 & 1.26 & 0.30 & 0.84 & 1.50 \\
$y_{i}(\mathrm{~m})$ & 0.75 & 0.73 & 0.55 & 0.63 & 0.21 & 0.65 & 0.06 & 0.24 & 0.62 & 0.80 & 0.40 & 0.50 \\
\hline
\end{tabular}

Table 2. $\left(x_{i}, y_{i}\right)$ co-ordinates of point excitation $i$. 